NASA/TM-2003-212219

Specific Hardening Function Definition and Characterization of a Multimechanism Generalized Potential-Based Viscoelastoplasticity Model

\author{
A.F. Saleeb \\ University of Akron, Akron, Ohio \\ S.M. Arnold \\ Glenn Research Center, Cleveland, Ohio
}


Since its founding, NASA has been dedicated to the advancement of aeronautics and space science. The NASA Scientific and Technical Information (STI) Program Office plays a key part in helping NASA maintain this important role.

The NASA STI Program Office is operated by Langley Research Center, the Lead Center for NASA's scientific and technical information. The NASA STI Program Office provides access to the NASA STI Database, the largest collection of aeronautical and space science STI in the world. The Program Office is also NASA's institutional mechanism for disseminating the results of its research and development activities. These results are published by NASA in the NASA STI Report Series, which includes the following report types:

- $\quad$ TECHNICAL PUBLICATION. Reports of completed research or a major significant phase of research that present the results of NASA programs and include extensive data or theoretical analysis. Includes compilations of significant scientific and technical data and information deemed to be of continuing reference value. NASA's counterpart of peerreviewed formal professional papers but has less stringent limitations on manuscript length and extent of graphic presentations.

- TECHNICAL MEMORANDUM. Scientific and technical findings that are preliminary or of specialized interest, e.g., quick release reports, working papers, and bibliographies that contain minimal annotation. Does not contain extensive analysis.

- CONTRACTOR REPORT. Scientific and technical findings by NASA-sponsored contractors and grantees.
- CONFERENCE PUBLICATION. Collected papers from scientific and technical conferences, symposia, seminars, or other meetings sponsored or cosponsored by NASA.

- SPECIAL PUBLICATION. Scientific, technical, or historical information from NASA programs, projects, and missions, often concerned with subjects having substantial public interest.

- TECHNICAL TRANSLATION. Englishlanguage translations of foreign scientific and technical material pertinent to NASA's mission.

Specialized services that complement the STI Program Office's diverse offerings include creating custom thesauri, building customized databases, organizing and publishing research results ... even providing videos.

For more information about the NASA STI Program Office, see the following:

- Access the NASA STI Program Home Page at http://www.sti.nasa.gov

- E-mail your question via the Internet to help@sti.nasa.gov

- Fax your question to the NASA Access Help Desk at 301-621-0134

- Telephone the NASA Access Help Desk at 301-621-0390

- Write to:

NASA Access Help Desk

NASA Center for AeroSpace Information 7121 Standard Drive

Hanover, MD 21076 
NASA/TM-2003-212219

Specific Hardening Function Definition and

Characterization of a Multimechanism

Generalized Potential-Based

Viscoelastoplasticity Model

\author{
A.F. Saleeb \\ University of Akron, Akron, Ohio \\ S.M. Arnold \\ Glenn Research Center, Cleveland, Ohio
}

National Aeronautics and

Space Administration

Glenn Research Center 


\section{Acknowledgments}

Professor Saleeb would like to acknowledge the financial support provided by NASA Glenn Research Center under Grant (NCC3-808) to the University of Akron to conduct this work.

Trade names or manufacturers' names are used in this report for identification only. This usage does not constitute an official endorsement, either expressed or implied, by the National Aeronautics and Space Administration.

Available from

NASA Center for Aerospace Information 7121 Standard Drive

Hanover, MD 21076
National Technical Information Service 5285 Port Royal Road Springfield, VA 22100

Available electronically at http:/ /gltrs.grc.nasa.gov 


\title{
Specific Hardening Function Definition and Characterization of A Multimechanism Generalized Potential-Based Viscoelastoplasticity Model
}

\author{
A. F. Saleeb \\ Department Civil Engineering \\ University of Akron \\ Akron, $\mathrm{OH} 44325$ \\ S. M. Arnold \\ National Aeronautics and Space Administration \\ Glenn Research Center \\ Cleveland $\mathrm{OH}, 44135$
}

\begin{abstract}
Given the previous complete-potential structure framework (see ARNOLD AND SALEEB [1994]), together with the notion of strain- and stress- partitioning in terms of separate contributions of several submechanisms (viscoelastic and viscoplastic) to the thermodynamic functions (stored energy and dissipation), see SALEEB ET AL. [2001], a detailed viscoelastoplastic multimechanism characterization of a specific hardening functional form of the model is presented and discussed. TIMETAL $21 \mathrm{~S}$ is the material of choice as a comprehensive test matrix, including creep, relaxation, constant strain-rate tension tests, etc. are available at various temperatures. Discussion of these correlations tests, together with comparisons to several other experimental results, are given to assess the performance and predictive capabilities of the present model particularly with regard to the notion of hardening saturation as well as the interaction of multiplicity of dissipative (reversible/irreversible) mechanisms.
\end{abstract}

\section{Nomenclature}

Invariants
$\Omega, \Omega_{R}, \Omega_{I R}$
$\Phi, \Phi_{R}, \Phi_{I R}$
$F$
$G^{(b)}$
$h_{b}$

Invariants complementary dissipation potential; R- reversiable, IR -irreversible

Gibb's complementary potential; R- reversiable, IR irreversible

Bingham-Prager threshold function

normalized second invariant function

invariant material function 


\section{Stresses}

$\sigma_{i j}$

$\left(\sigma_{s}\right)_{i j}$

$q_{i j}$

$\sigma_{i j}-\alpha_{i j}$

$\alpha_{i j}$

$\kappa$

$\kappa_{b}$

\section{Strains}

$\epsilon_{i j}, \epsilon_{i j}^{v e}, \epsilon_{i j}^{v p}, \epsilon_{i j}^{t h}$

$p_{i j}$

$\gamma_{i j}$

Material Parameters

$E_{i j k l}, M_{i j k l}^{(a)}, \eta_{i j}^{(a)}$

$H_{(b)}, R_{(b)}$

$\mu$

$E_{s}, E_{m}^{(a)}$

$n, \beta, m$

$r\left((G)^{(b)}\right), h\left(G^{(b)}\right), f(F)$

$\rho_{a}$

$\nu$

\section{Miscellaneous}

$\mathcal{M}_{i j k l}$

$N_{i j k l}$

$\delta_{i j}$

\langle\rangle

$H v[]$

$(\cdot)$

M

$N$
Applied Cauchy stress tensor

viscoelastic equilibrium stress tensor

viscoelastic, non-equilibrium stress

viscoplastic equilibrium stress tensor

viscoplastic non-equilibrium stress tensor

drag stress

either a normalizing stress or hardening threshold stress per mechanism

total, reversable (viscoelastic), irreversible (viscoplastic) and thermal strain tensors, respectively internal reversible strain tensor (displacement-like) internal irreversible strain tensor

reversible elastic stiffness, viscoelastic stiffness and viscosity coefficient tensor for each $\mathrm{a}^{\text {th }}$ mechanism, respectively hardening and thermal recovery material parameters per mechanism

material parameter associated with the irreversible viscosity of the material

denotes the elastic stiffness and maxwell spring stiffness per $\mathrm{a}^{\text {th }}$ mechanism, respectively

material exponents

material functions

relaxation time for the $\mathrm{a}^{t h}$ mechanism; $=E_{\eta}^{(a)} / E_{m}^{(a)}$

Possion ratio

Devatoric operator (isotropic or anisotropic)

Isotropic directionality tensor

Kronecker delta function

Macauley bracket

Heaviside unit function

time derivative (or rate) notation

number of reversible mechanisms

number of irreversible mechanisms 


\section{Introduction}

Extensive research efforts have been made over the years on the phenomenological representations of material behavior. Significant progress has been made in the development of theories of viscoelasticity and viscoplasticity for the modeling of inelastic properties. In particular, the present state-of-the-art in mathematical modeling of viscoplastic deformation of metals is very well developed; i.e., based on the so-called internal variable formalism in thermodynamics of irreversible processes (COLEman\&GurTin [1967], Lubliner [1973], Lemaitre\&Chaboche[1990], Chaboche[1989], Arnold\&Saleeb [1994], Arnold et. al. [1994a,1995], FREED\&WALKER[1993]).A larger number of specialized forms of unified viscoplastic models (e.g., isotropic or anisotropic, fully associative or nonassociative, etc.) have been successfully applied in recent years to different high-temperature metals, alloys, and composite material systems (Lemaitre\&Chaboche[1990], Arnold et. Al. [1994a,1995], Freed\&Walker[1993], Krausz\&Krausz[1996], Hart [1976], Hui [1985], Kumar et al. [1980] and SALEEB\&WILt [1993]). In the present paper, we continue along our previous efforts, and utilize the fully associative generalized viscoelastoplastic potential framework ${ }^{1}$ (Arnold\&S aleeb [1994], Arnold et. Al. [1994a,b;1995], Saleeb\&Wilt [1993] and Saleeb ET AL. [2001]) to represent a rather comprehensive set of experimental results performed on the titanium alloy TIMETAL 21S. TIMETAL $21 \mathrm{~S}$ is a $\beta$-titanium alloy with a nominal composition of Ti-15Mo-3NB-3Al-0.2Si (\% weight). Depending on the stress/deformations levels, this material was found to exhibit both reversible and irreversible behavior with marked rate dependency in each regime.

The objective of the present paper is twofold: the first being an evaluation of a specific form for the material hardening function that has a true saturation value (or limit state) associated with it, and the second is the characterization and evaluation of model representations with multiple mechanism present in both the reversible and irreversible portions of the model, given the above mentioned material system. The coupled multimechanism viscoelastoplastic model (see SALEEB ET AL. [2001]) employed has been formulated to allow for sufficient generality in its potential functions (Gibb's and dissipation) through a systematic introduction of several mechanisms (internal state variables of the tensorial type) for both viscoelastic and viscoplastic response components. With the viscoelastic part, utilizing the concept of an equilibrium stress, leading to rate dependency upon instantaneous loading, as well as to a unique limiting state of elastic deformation at infinite times. The viscoplasticity formulation accounts for both nonlinear kinematic hardening and static-recovery mechanisms. We have previously reported (SALEEB ET AL. [2001]) on the implicit numerical-integration aspects, as well as the computational aspects concerning the automation of material-parameter-estimation procedures using the software called COMPARE (COnstitutive Material PARameter Estimator). We refer the reader to the above paper (and other references therein) for further details.

This paper begins in section 2, with a brief outline of the general fully associative framework for several interacting mechanisms of the viscoelastic and viscoplastic types, leading to the governing flow and evolutionary rate equations. The specific functional forms describing the hardening and thermal recovery of the material are then described. In section 3 , the general

\footnotetext{
${ }^{1}$ This framework falls into the category of phenomenological (or macroscopic) material representation based on continuum mechanics and internal state variables and has been labeled by us as belonging to the GVIPS (Generalized VIscoelastoplastic with Potential Structure) class of material models.
} 
characterization strategy is discussed with the specific values for the various material parameters, given a single, two and three mechanism viscoplastic (irreversible) representation. The six mechanism viscoelastic (reversible) portion of the model (taken from previous work) is also repeated within for convenience. This is then followed by section 4 which describes in detail the results of the comparison of saturating versus non-saturating material hardening functional forms of the model and the correlative and predictive capability of multimechanism representations (wherein multiple viscoplastic internal material clocks are accounted for) verses a single viscoplastic representation (that only admits a single internal clock).

\section{Complete Potential Structure}

We begin by summarizing the basic equations governing the behavior of a material element. The discussion is limited to the case of small deformations and isothermal conditions, in which the initial (virgin) state is assumed to be stress free (as well as, all initial values of internal state parameters are assumed zero) throughout. Here, and for the remainder of the paper, a Cartesian reference frame is utilized, along with index notation (wherein summation is implied for repeated subscripts). However, for convenience, we also utilize superscript letters placed between parentheses as indices to identify sets of internal state parameters and when needed the summation over these will be indicated explicitly by the summation symbol.

All equations are written for the general 3D (three-dimensional) case; but the forms are directly applicable in subspaces (e.g., 2D plane-stress or plane-strain, etc.); see (SALEEB\&WILT [1993], SaleEB ET AL. [1998;1999]). For conciseness, we define the total number of dissipative viscoelastic state variables (each of the second-order tensor type) as $M$; i.e.; the corresponding non-equilibrium stress tensors (SAleEb\&ARnold[1997] and ARnold ET. AL. [1997]) are

$\mathbf{q}_{i j}^{(a)}(a=1,2, \ldots M)$ and their associated conjugate (strain-like) tensors are $\mathbf{p}_{i j}^{(a)}$. In addition, we define the equilibrium stress tensor $\left(\boldsymbol{\sigma}_{s}\right)_{i j}$ and its strain conjugate as $\varepsilon_{i j}^{v e}$. Similarly, for the viscoplastic mechanisms, we use the notation $\boldsymbol{\alpha}_{i j}^{(b)}(b=1,2, \ldots, N)$ for the back stresses (kinematic hardening) and $\gamma_{i j}{ }^{(b)}$ for their conjugate or dual (strain-like) variables, for a total of $N$ viscoplastic (second-order) tensorial state variables.

\subsection{Potentials and State Equations}

In accordance with the basic hypothesis of the additive decomposition of total strain tensor, $\varepsilon_{i j}$, into three components, that is a reversible (i.e., elastic/viscoelastic), $\varepsilon_{i j}^{v e}$; an irreversible (i.e., viscoplastic), $\varepsilon_{i j}^{v p}$; and a thermal strain, $\varepsilon_{i j}^{t h}$, component; we have:

$$
\varepsilon_{i j}=\varepsilon_{i j}^{v e}+\varepsilon_{i j}^{v p}+\varepsilon_{i j}^{t h}
$$

Similarly, an additive decompositon for the two fundamental potentials, that is, the Gibbs, complementary function, $\Phi$, and dissipation function, $\Omega$, are assumed:

$$
\begin{gathered}
\Phi\left(\boldsymbol{\sigma}_{i j}, \boldsymbol{\alpha}_{i j}^{(b)}, \mathbf{q}_{i j}^{(a)}\right)=\Phi_{R}\left(\boldsymbol{\sigma}_{i j}, \mathbf{q}_{i j}^{(a)}\right)+\Phi_{I R}\left(\boldsymbol{\alpha}_{i j}^{(b)}\right) \\
\Omega\left(\boldsymbol{\sigma}_{i j}, \boldsymbol{\alpha}_{i j}^{(b)}, \mathbf{q}_{i j}^{(a)}\right)=\Omega_{R}\left(\mathbf{q}_{i j}^{(a)}\right)+\Omega_{I R}\left(\left(\boldsymbol{\sigma}_{i j}-\boldsymbol{\alpha}_{i j}\right), \boldsymbol{\alpha}_{i j}^{(b)}\right)
\end{gathered}
$$


where the functional dependencies on the external $\left(\boldsymbol{\sigma}_{i j}\right)$ and internal $\left(\mathbf{q}_{i j}^{(a)}\right.$ and $\left.\boldsymbol{\alpha}_{i j}^{(b)}\right)$ state variables, with $(a=1,2, \ldots, M)$ and $(b=1,2, \ldots, N)$, and the conjugate variables $\varepsilon_{i j}^{v p}$ (also $\varepsilon_{i j}$ ), $\varepsilon_{i j}^{v e}$ (also $\mathbf{p}_{i j}^{(a)}$ ), and $\boldsymbol{\gamma}_{i j}^{(b)}$, respectively, are given by the following equations of state:

$$
\begin{gathered}
\boldsymbol{\varepsilon}_{i j}-\boldsymbol{\varepsilon}_{i j}{ }^{v p}=\frac{\partial \Phi_{R}}{\partial\left(\boldsymbol{\sigma}_{s}\right)_{i j}} \\
\boldsymbol{\varepsilon}_{i j}^{v e}-\mathbf{p}_{i j}^{(a)}=\frac{\partial \Phi_{R}}{\partial \mathbf{q}_{i j}^{(a)}} \\
\gamma_{i j}^{(b)}=\frac{\partial \Phi_{I R}}{\partial \boldsymbol{\alpha}_{i j}^{(b)}}
\end{gathered}
$$

The corresponding rate forms are then

$$
\begin{gathered}
\dot{\boldsymbol{\varepsilon}}_{i j}-\dot{\boldsymbol{\varepsilon}}_{i j} v p=\frac{d}{d t}\left(\frac{\partial \Phi_{R}}{\partial\left(\boldsymbol{\sigma}_{s}\right)_{i j}}\right)=\frac{\partial^{2} \Phi_{R}}{\partial\left(\boldsymbol{\sigma}_{s}\right)_{i j} \partial\left(\boldsymbol{\sigma}_{s}\right)_{k l}}\left(\dot{\boldsymbol{\sigma}}_{s}\right)_{k l} \\
\dot{\varepsilon}_{i j}{ }^{v e}-\dot{\mathbf{p}}_{i j}^{(a)}=\frac{d}{d t}\left(\frac{\partial \Phi_{R}}{\partial \mathbf{q}_{i j}^{(a)}}\right)=\frac{\partial^{2} \Phi_{R}}{\partial \mathbf{q}_{i j}^{(a)} \partial \mathbf{q}_{k l}^{(a)}} \dot{\mathbf{q}}_{k l}^{(a)} \\
\dot{\gamma}_{i j}^{(b)}=\frac{d}{d t}\left(\frac{\partial \Phi}{\partial \boldsymbol{\alpha}_{i j}^{(b)}}\right)=\frac{\partial^{2} \Phi_{I R}}{\partial \boldsymbol{\alpha}_{i j}^{(b)} \partial \boldsymbol{\alpha}_{k l}^{(b)}} \dot{\boldsymbol{\alpha}}_{k l}^{(b)} ; \quad \text { or } \quad \dot{\boldsymbol{\alpha}}_{i j}^{(b)}=\left[\frac{\partial^{2} \Phi_{I R}}{\partial \boldsymbol{\alpha}_{i j}^{(b)} \partial \boldsymbol{\alpha}_{k l}^{(b)}}\right]^{-1} \dot{\gamma}_{k l}^{(b)}
\end{gathered}
$$

where the above equation is the internal constitutive rate equations for the internal (nonequilibrium) state variable for each hardening mechanism.

The corresponding flow and evolution (rate) equations are similarly obtained from the dissipation function and are as follows:

$$
\begin{gathered}
\dot{\varepsilon}_{i j}{ }^{v p}=\frac{\partial \Omega_{I R}}{\partial \boldsymbol{\sigma}_{i j}} \\
\dot{\gamma}_{i j}{ }^{(b)}=\frac{-\partial \Omega_{I R}}{\partial \boldsymbol{\alpha}_{i j}^{(b)}} ; \quad b=1,2, \ldots, N \\
\dot{\mathbf{p}}_{i j}{ }^{(a)}=\frac{\partial \Omega_{R}}{\partial \mathbf{q}_{i j}^{(a)}} ; \quad a=1,2, \ldots, M
\end{gathered}
$$

Note in Eqn. (5) only a single viscoelastic strain component is associated with all viscoelastic stress components. Also for the viscoelastic response, the total stress is decomposed into an equilibrium stress, $\left(\boldsymbol{\sigma}_{s}\right)_{i j}$, and a non-equilibrium (dissipative) reversible stress, $\mathbf{q}_{i j}$; i.e.,

$$
\left(\boldsymbol{\sigma}_{s}\right)_{i j}=\boldsymbol{\sigma}_{i j}-\mathbf{q}_{i j} ; \quad \text { where } \mathbf{q}_{i j}=\sum_{a=1}^{M} \mathbf{q}_{i j}^{(a)}
$$

and for the viscoplastic response, the total stress is decomposed again into an equilibrium, $\left(\boldsymbol{\sigma}_{i j}-\boldsymbol{\alpha}_{i j}\right)$, and non-equilibrium, $\boldsymbol{\alpha}_{i j}$, component; where 


$$
\boldsymbol{\alpha}_{i j}=\sum_{b=1}^{N} \boldsymbol{\alpha}_{i j}^{(b)}
$$

The above GVIPS framework attempts to capture the underlying physical processes associated with microscopic defects (e.g. dislocations, grain boundaries, voids etc. in metals) and their complicated interactions which span an entire spectrum of length scales (i.e. from the "atomistic (nano) - microscale", to the "mesoscale" to the final "macroscale") by introducing the notion of multiplicity of mechanisms in the mathematical description. These mechanisms, reflecting the vastly different interactions, give rise to the introduction of an aggregate of individual internal state variables (i.e., $\mathbf{q}_{i j}^{(a)}$ and $\boldsymbol{\alpha}_{i j}^{(b)}$ ); e.g. many tensors each accounting for interactions at different length scales, that is, "short" range between dislocations and other dislocations, and "long" range between a well developed dislocation cell or subgrain and other defects and interfaces. In addition to the differences in "microstructural" length scales, the time rates of change governing their dynamics are also known to be vastly different, hence the notion of multiple (or spectrum of) characteristic relaxation times in the GVIPS formulation. i.e., $\mathbf{M}_{i j k l}^{(a)}$ and $\boldsymbol{\eta}_{i j k l}^{(a)}$ of Eqns.(15) and (17) . Furthermore, with energy measure providing the major consideration in dislocation dynamic theories (e.g., in studying thermal activation, mass and vacancies diffusion, energy barrier such as jog formation energy, rate-dependent and multi-species (distributions of finite-strength obstacles, etc.)) and the intricate interaction of such "unit" processes, with their vastly different energy contents and rate-limiting values, the partitioning of the overall supplied work into energy storage (e.g. hardening) and energy dissipation (e.g. recovery and inelastic flows) components that proceed with varying degrees of competition during the deformation of the material became a motivating factor, see Eqns. (2) and (3) - and more specifically Eqns. (15) through (18). Hence the emphasis on complete ${ }^{2}$-potential "energy" structure which leads to a "hierarchy" of representations, from the Gibb's and dissipation functions (Grandparent), to the kinematic decomposition of the deformation and microstructure state equations (Parent), to the kinetic and evolution equations (Children). This structure then guarantees i) that the overall response is always bounded in terms of the total and/or rate quantities (e.g. transient to steady state creep, or from a state of nonlinear hardening to a state of saturation in hardening) and ii) the availability of symmetric tangent stiffness matrices which greatly enhance computational robustness. Finally, the use of multimechanisms (embedding the effects of many time/length scales) in the GVIPS class of formulation enables the specialization of this general model into simpler (more restricted in scope) formulations, e.g. purely elastic, linear viscoelastic, classical rate-independent elastoplastic, as well as more elaborate forms of hereditary descriptions (involving viscous effects, nonlinear hardening, dynamic recovery, thermal/static recovery, relaxation, ratchetting or shakedown phenomena under load cycles) to name a few.

\subsection{Specific Functional Forms:}

For concreteness, we summarize here the specified functional forms that have been used in our previous work (ARnold et. AL. [1994a,b;1995], Saleeb\&Wilt [1993], SaleeB\&ARnold[1997],

\footnotetext{
${ }^{2}$ By complete we mean a formulation, as described by Eqns. (2) -(12), whereby the flow and evolutionary equations are fully-associative (are normal to the same dissipation potential surface).
} 
Arnold Et. AL. [1997] and Saleeb Et. AL. [2001]) and will be used for the most part here. These forms are quadratic for the viscoelastic contribution and essentially the same "powertype" nonlinear forms previously used for the viscoplasticity functions $\bar{H}_{(b)}, \Omega_{1}$, and $\Omega_{2}^{(b)}$ in terms of the invariants of their respective arguments $\boldsymbol{\alpha}_{i j}^{(b)},\left(\boldsymbol{\sigma}_{i j}-\boldsymbol{\alpha}_{i j}\right)$ and $\boldsymbol{\alpha}_{i j}^{(b)}$, see eqns. 16, 18 and 20 below. However, a new functional form, $h\left(G^{(b)}\right)$, for the hardening function will be introduced and investigated as well.

The specific forms for the reversible and irreversible potentials and driving functions are as follows:

$$
\begin{gathered}
\Phi_{R}=\Phi_{R}\left(\boldsymbol{\sigma}_{i j}, \mathbf{q}_{i j}^{(a)}\right)=\frac{1}{2}\left(\boldsymbol{\sigma}_{s}\right)_{i j} \mathbf{E}_{i j k l}^{-1}\left(\boldsymbol{\sigma}_{s}\right)_{k l}+\frac{1}{2} \sum_{a=1}^{M} \mathbf{q}_{i j}^{(a)}\left[\mathbf{M}_{i j k l}^{(a)}\right]^{-1} \mathbf{q}_{k l}^{(a)}+\sum_{a=1}^{M} \mathbf{q}_{i j}^{(a)} \mathbf{p}_{i j}^{(a)} \\
\Phi_{I R}=\Phi_{I R}\left(\boldsymbol{\sigma}_{i j}, \boldsymbol{\alpha}_{i j}^{(b)}\right)=\boldsymbol{\sigma}_{i j} \varepsilon_{i j}^{v p}+\sum_{b=1}^{N} \bar{H}_{(b)}\left(G^{(b)}\right)
\end{gathered}
$$

and

$$
\begin{aligned}
\Omega_{R} & =\frac{1}{2} \sum_{a=1}^{M} \mathbf{q}_{i j}^{(a)}\left[\boldsymbol{\eta}_{i j k l}^{(a)}\right]^{-1} \mathbf{q}_{k l}^{(a)} \\
\Omega_{I R} & =\Omega_{1}(F)+\sum_{b=1}^{N} \Omega_{2}^{(b)}\left(G^{(b)}\right)
\end{aligned}
$$

where

$$
\begin{gathered}
F=\frac{1}{2 \kappa^{2}}\left(\boldsymbol{\sigma}_{i j}-\boldsymbol{\alpha}_{i j}\right) \mathcal{M}_{i j k l}\left(\boldsymbol{\sigma}_{k l}-\boldsymbol{\alpha}_{k l}\right)-1 \\
G^{(b)}=\frac{1}{2 \kappa_{(b)}^{2}}\left(\boldsymbol{\alpha}_{i j}^{(b)} \mathcal{M}_{i j k l} \boldsymbol{\alpha}_{k l}^{(b)}\right)
\end{gathered}
$$

and the required partial derivatives are:

$$
\frac{1}{\kappa^{2}} \frac{\partial \Omega_{1}}{\partial F}=f(F)=\frac{F^{n}}{2 \mu} \quad \frac{1}{\kappa_{(b)}^{2}} \frac{\partial \Omega_{2}^{(b)}}{\partial G^{(b)}}=\frac{r\left(G^{(b)}\right)}{h\left(G^{(b)}\right)} \quad \frac{1}{\kappa_{(b)}^{2}} \frac{\partial \bar{H}_{(b)}}{\partial G^{(b)}}=\frac{1}{h\left(G^{(b)}\right)}
$$

Note in the above, $\mathbf{E}_{i j k l}$ and $\mathbf{M}_{i j k l}$ are fourth-order tensors of viscoelastic stiffness moduli (with $\mathbf{E}_{i j k l}^{-1}$ and $\left[\mathbf{M}_{i j k l}^{(a)}\right]^{-1}$ being the corresponding compliance tensors $) ; \boldsymbol{\eta}_{i j k l}^{(a)}(a=1,2, \ldots, M)$ are the fourth-order tensorial viscosity coefficients associated with the $a^{\text {th }}$ dissipative viscoelastic mechanism; the $\Omega_{1}$, and $\Omega_{2}^{(b)}$ are the inelastic dissipations due to plastic hardening and static (thermal) recovery, respectively; and each $\bar{H}_{(b)}$ is taken to be a nonlinear function of the corresponding viscoplastic internal state tensor $\boldsymbol{\alpha}_{i j}^{(b)}$. To emphasize the functional dependency in the potentials, e.g., $\Phi_{R}, \Phi_{I R}$ and $\bar{H}_{(b)}$, we have shown the corresponding arguments in parentheses.

The only remaining functions that need to be assumed are those defining the competitive processes within a material, that is the hardening and recovery terms. The function that drives the thermal recovery is taken as previously to be a "power-type" function, that is, 


$$
r\left(G^{(b)}\right)=R_{(b)}\left[G^{(b)}\right]^{m_{(b)}}
$$

whereas two related but distinct forms for the hardening function, $h\left(G^{(b)}\right)$, will be assumed and investigated herein. The first nonsaturating hardening form, which has been used extensively in the past (e.g., Robinson [1978] and Arnold Et. Al. [1994a,b and 1995] and Saleeb ET.AL. [2001]),

$$
h_{\text {nonsat }}\left(G^{(b)}\right)=\frac{H_{(b)}}{\left[G^{(b)}\right]^{\beta_{(b)}}}
$$

is also of the power type. This form, although nonlinear in nature, is however, deficient in two ways; i) it is unable to ever reach a true limit state (unless the constant $\beta$ is unrealistically high) in which hardening would cease, i.e., $h(G)=0$, and ii) no physical interpretation can be associated with the "normalizing" stress, $\kappa_{(b)}$, for each individual mechanism. Consequently, an alternative form which explicitly posses a limit state, irrespective of the exponent $\beta$, can be introduced, that is:

$$
h_{\text {sat }}\left(G^{(b)}\right)=H_{(b)}\left\langle 1-\sqrt{G^{(b)}}\right\rangle^{\beta_{(b)}}
$$

Note that with this form the function $h(G)$ will now always go to zero, for a given mechanism, whenever the now explicit hardening threshold, $\kappa_{(b)}$, is exceeded. Thus this new form can simulate saturation of a tensile curve (particularly difficult at lower temperatures where thermal recovery is naturally supressed) while still accurately representing other loading histories, e.g., creep and relaxation. A comparison of the respective capabilities of these forms will be discussed in more detail in section 5.1. Note also that, though different in their behaviors, both forms in Eqns. 23 and 24 involve the same number of material constants, thus no additional price is paid for introducing saturation.

Finally, in the above, $\kappa, \mu, n$ represent viscoplastic flow material constants, whereas the $H_{(b)}, \beta_{(b)}$ are hardening material constants; $R_{(b)}, m_{(b)}$ are recovery material constants; and constants $\kappa_{(b)}$ are either "normalizing" or hardening threshold stresses, for the individual mechanism $(b)$, where $b=1,2, \ldots N$, depending upon the form of the hardening function, $h\left(G^{(b)}\right)$, assumed.

\subsection{Physical, Micromechanical Motivation}

There are a number of observations, both from the viewpoints of physical micromechanical origins as well as the macroscopic manifestations in experiments, which provide strong motivation for adopting expressions such as in Eqn. (24). For example, dislocations act as the principle carriers of plastic deformations in metals. According to modern theories of dislocation dynamics [HIRTH\&Lothe [1982]], there are numerous primary and secondary interactions affecting dislocation mobility (e.g., among dislocations with other dislocations, or between dislocations and other obstacles/defects/ or internal and free-boundaries, etc.). The tendency of this motion is towards more of a modulated substructure, i.e., dislocation cells and subgrains, as well as heterogeneous patterns associated with slip bands, microshear bands, persistent slip bands and dislocation tangles. To define these dislocation arrangements, there is a need to use 
a large number of parameters (e.g., in addition to dislocation densities, more irregular statistical distributions in the form of higher-order correlation functions are also required). However, despite the inherent complexity in describing all the details of relevant elementary mechanisms and denominate unit processes operating at the microscale, most studies [ZBIB ET AL.[2002], Arsenlis\&PARK[2002] and StAinier ET AL. [2002]] on the factors affecting the rate of dislocation motion support the notion of a competing nature. That is, one distinguishes between those mechanisms that hinder the motion (e.g., dislocations generation in pile-ups) and those which facilitate the motion (such as the increased mobility of existing dislocations and/or annihilation of dislocations by climb), wherein the macroscopic forces driving these competing unit processes are the applied stresses, the loading or deformation rates, and the temperature. For example, the above generation-annihilation formulation for a discrete set of eighteen dislocation densities were utilized as internal state parameters in ARSENLIS\&PARK [2002] to describe the state of crystallographic hardening in crystal plasticity.

On the other hand, recent three-dimensional simulations based on dislocation dynamics [ZBIB ET AL, 2002] have clearly demonstrated that when the dislocation sources start operating, the dislocation density increases sharply at the beginning, but then the rate of increase diminishes gradually afterwards, until a saturation value is finally reached. At this latter condition of saturation, the portion of the dislocation segments generated by the sources are equalized by the dislocation segments disappearing on interaction with the free surfaces and by dislocation -dislocation annihilation. This saturation state was consistently observed at all deformation rates considered; the differences being the attainment of higher dislocation densities at the higher strain rates. Similarly, recent investigations on the micromechanical basis/multiscale modeling of deformation in bcc crystals [STAINIER ET AL. [2002]] have also resulted in observing saturation states of hardening at sufficiently large strains, with the marked dependency of the saturation state of stress on temperature and strain rates. The analysis considered an elaborate set of microscopic processes; i.e., double kink formation and thermally-activated motion of kinks; short-range interactions between primary and forest dislocation species (with different obstacle strength); dislocation multiplication due to breeding by double cross-slip as well as fixed Frank-Read sources; and dislocations pair annihilation.

Alternatively, macroscopic scale experiments on metals, such as described in the later sections, exhibit saturation stress states in the fully developed plastic region. This often occurs under a wide variety of (constant) strain rates, with a higher saturation stress resulting from the increased loading rates. In summary, both microscopically as well as macroscopically, there is ample evidence for the existence of states of hardening saturation. This is the main motivation for adopting such a functional form as that proposed in Eqn. (24).

Finally, there are important differences in the present treatment of the saturation phenomena, as compared to existing alternatives in the literature (e.g. Lemaitre And Chaboche [1990], Freed [1993], Kremple [1996] and Ho And Kremple [2002]). More specifically, we do not introduce such notions as explicit bounding surfaces to limit the stress magnitudes,or the idea of overstress/equilibrium versus hardening stress, or the separation of the material's inelasticity into creep plus perfectly-plastic strains. In addition, our treatment indicates that hardening-stauration is always possible, irrespective of the rates of continued loadings, thus emphasizing the physical evidence that the internal structure will ultimately reach a stable/modulated microstructural configuration (of course this is true without the intervention of other "unstable" phenomena, such as damage and failures, which are outside the scope of this paper). 


\subsubsection{Resulting Multiaxial Flow and Evolution Equations}

With the selection of the particular functional forms above, the present viscoelastoplastic model is now complete within the context of a fully associative potential structure. The following expressions constitute the governing associated flow and evolution equations, assuming multiaxial anisotropic behavior.

$$
\begin{gathered}
\dot{\boldsymbol{\sigma}}_{i j}=\mathbf{E}_{i j k l}\left(\dot{\boldsymbol{\varepsilon}}_{k l}-\dot{\boldsymbol{\varepsilon}}_{k l}^{v p}\right)+\sum_{a=1}^{M} \dot{\mathbf{q}}_{i j}^{(a)} \\
\dot{\mathbf{q}}_{i j}^{(a)}=\mathbf{M}_{i j k l}\left(\dot{\boldsymbol{\varepsilon}}_{k l}-\dot{\boldsymbol{\varepsilon}}_{k l}^{v p}\right)+\mathbf{M}_{i j k l} \boldsymbol{\eta}_{k l r s}^{-1} \mathbf{q}_{r s}^{(a)} \\
\dot{\varepsilon}_{i j}^{v p}=f(F) \boldsymbol{\Gamma}_{i j} \quad \text { if } \quad F \geq 0 \\
\dot{\boldsymbol{\varepsilon}}_{i j}^{v p}=0 \quad \text { otherwise } \\
\dot{\boldsymbol{\alpha}}_{i j}^{(b)}=\mathbf{Q}_{i j k l}^{(b)}\left[\dot{\varepsilon}_{k l}^{v p}-\frac{r\left(G^{(b)}\right)}{h\left(G^{(b)}\right)} \boldsymbol{\pi}_{k l}^{(b)}\right] \quad \text { if } \boldsymbol{\pi}_{k l}^{(b)} \cdot\left(\boldsymbol{\sigma}_{k l}-\alpha_{k l}\right) \geq 0
\end{gathered}
$$

where

$$
\begin{gathered}
\mathbf{Q}_{i j k l}^{(b)}=\left[\frac{\partial^{2} \bar{H}_{(b)}}{\partial \boldsymbol{\alpha}_{i j}^{(b)} \partial \boldsymbol{\alpha}_{k l}^{(b)}}\right]^{-1}=h\left(G^{(b)}\right)\left[\mathbf{Z}_{m}+\frac{h^{\prime}\left(G^{(b)}\right)}{h\left(G^{(b)}\right)\left[1-\frac{h^{\prime}\left(G^{(b)}\right)}{h\left(G^{(b)}\right)}\left(2 \kappa_{(b)}^{2} G^{(b)}\right)\right]} \boldsymbol{\alpha}_{i j}^{(b)} \boldsymbol{\alpha}_{k l}^{(b)}\right] \\
\boldsymbol{\Gamma}_{i j}=\mathcal{M}_{i j k l}\left(\boldsymbol{\sigma}_{k l}-\boldsymbol{\alpha}_{k l}\right) ; \quad \boldsymbol{\pi}_{k l}^{(b)}=\mathcal{M}_{k l i j} \boldsymbol{\alpha}_{i j}^{(b)} \\
\mathbf{q}_{i j}=\sum_{a=1}^{M} \mathbf{q}_{i j}^{(a)} ; \quad \boldsymbol{\alpha}_{i j}=\sum_{b=1}^{N} \boldsymbol{\alpha}_{i j}^{(b)}
\end{gathered}
$$

and

$$
h^{\prime}(\cdot)=\frac{1}{\kappa_{(b)}^{2}} \frac{\partial h(\cdot)}{\partial G^{(b)}}
$$

wherein $h(\cdot)$ becomes either $h_{\text {old }}$ or $h_{\text {new }}$ depending upon the specific form in view. Note $\mathbf{Z}_{m}$ is the "generalized" inverse of $\mathcal{M}_{i j k l}$; see SALEEB\&WiLt [1993] for further elaboration on this. Details regarding the numerical solution of these equations have been discussed previously, see Saleeb\&Wilt [1993], Saleeb et al. [2001]. Furthermore, note that the flow and evolutionary loading conditions (Eqns. 27 and 28) are slightly modified as compared to this previous work, due to our recent consideration of multiaxial ratchetting behavior. These differences will be further elaborated upon in a future publication. 


\section{Model Characterization}

\subsection{Outline of the General Strategy}

The most important, and often times most difficult, aspect of modeling the behavior of a given material at elevated temperature is obtaining the required material functions, e.g., $f(F)$ and $g(G)$, for viscoplasticity and associated material parameters. The difficulty associated with this process typically stems from not only the variety in mathematical forms for the material functions (e.g., power law, exponential, hyperbolic sine, etc.), but also the fact that given the material functions there is no unique set of material parameters for any given load path. Therefore, numerous iterations and difficult compromises are required before a final set of material parameters (for the assumed material functions) can be obtained.

Traditionally, characterization has been accomplished through basic trial and error procedures (i.e., graphical and/or mechanistic) which attempt to fit the predicted response from the constitutive model to that response exhibited by the experimental test data. These approaches, however, are rather limited, difficult, and many times less than fruitful for more general and sophisticated constitutive models. This is particularly true when dealing with models that possess a very large number ${ }^{3}$ of material constants that; (i) are often lacking in their direct physical interpretation (not the case in the present model), (ii) may have vastly different magnitudes, and (iii) are highly interactive with each other. Further complications also arise when a large number of experimental tests of various types (i.e., stress-, strain-, or mixed-control) under transient and/or steady-state conditions, are expected to be simulated.

To overcome these difficulties, software tool (i.e., COMPARE) has been developed to enable a design engineer to easily and efficiently bridge this gap between constitutive theory and experimental test data, in which optimum material parameters are determined by minimizing the errors between experimental data and the correlated responses (SAlEEB ET AL. [2001] and [2002]). Within COMPARE the estimation of material parameters is cast as a minimumerror, weighted least-squares, multi-objective optimization problem; wherein, this optimization problem is solved using the Sequential Quadratic Programing Technique. COMPARE is sufficiently general to handle a comprehensive set of test data, under arbitrary load-control variables, multiaxial stress/strain state, and transient as well as steady-state response measurements.

\subsection{Characterization of Material Parameters}

Building upon our previous work [Arnold et. Al. [1994a,b], Saleeb\&Arnold[1997], and ARNold ET AL. [1997] ], a primary objective in this paper is to specify material functions and characterize the corresponding material parameters for the current multimechanism, viscoelastoplastic model of the GVIPS class with kinematic hardening; given TIMETAL $21 \mathrm{~S}^{4}$, an advanced titanium-based matrix commonly used in TMCs. Previous work (ARNOLD ET. AL. [1994a,b: 1997, 2000], SALEeB ET AL [2001] ) indicated that six viscoelastic mechanisms (i.e., $\mathrm{M}=6$ ) were sufficient to adequately span the entire reversible load spectrum with the proper rate sensitivity at $650^{\circ} \mathrm{C}$ and that a single viscoplastic transient mechanism (i.e., $\mathrm{N}=1$ ) appeared insufficient to accurately (quantitatively) represent the irreversible domain over a wide stress

\footnotetext{
${ }^{3}$ In the specific model presented, the total number of material parameters required are $5+5 \mathrm{~N}+2 \mathrm{M}$ where $\mathrm{N}$ defines the number of viscoplastic mechanisms and $M$ the number of viscoelastic.

${ }^{4}$ TIMETAL $21 \mathrm{~S}$ is a registered trademark of TIMET, Titanium Metals Corporation.
} 
range. Here we will expand upon this previous work by 1) comparing the simulation capabilities of the two viscoplastic hardening forms given in Section 3.2 (given a single viscoplastic mechanism), and 2) upon proper down selection of the material hardening function, establish the number of required viscoplastic submechanisms required to accurately simulate the irreversible deformation behavior of TIMETAL $21 \mathrm{~S}$ at $650^{\circ} \mathrm{C}$. In all cases considered herein, the number of viscoelastic mechanisms are held fixed at six, with the 14 reversible material constants being those determined previously [see Arnold et al. [2001]] and given in Table 1. Note a key assumption is that Poisson ratio's is identical in all three matrices $\mathbf{E}_{i j k l}, \mathbf{M}_{i j k l}^{(a)}$ and $\boldsymbol{\eta}_{i j k l}^{(a)}$; thus making the forth-order tensor moduli coaxial and given the assumption of material isotropy, these moduli take on the following form: $\mathbf{E}_{i j k l}=\mathrm{E}_{s} \mathbf{N}_{i j k l}, \mathbf{M}_{i j k l}^{(a)}=\mathrm{E}_{m}^{(a)} \mathbf{N}_{i j k l}$ and $\boldsymbol{\eta}_{i j k l}^{(a)}=\rho_{a} \mathbf{M}_{i j k l}^{(a)}$; where

$$
N_{i j k l}=\left\{\frac{\nu}{(1+\nu)(1-2 \nu)} \delta_{i j} \delta_{k l}+\frac{1}{(1+\nu)}\left(\delta_{i k} \delta_{j l}+\delta_{i l} \delta_{j k}\right)\right\}
$$

With respect to the irreversible domain $3+5 \mathrm{M}$ constants are required in total; where three ( i.e., $\kappa, \mu$, and $n$ ) are associated with the flow law which is driven by the equilibrium (effective) stress, and the remaining 5 per submechanism (three with the nonlinear hardening operator; i.e., $H_{b}, \beta_{b}$ and $\kappa_{b}$, the hardening threshold, and two with the thermal recovery term; i.e., $R_{b}$ and $m_{b}$ ) are associated with the evolution of the nonequilibrium (back or internal) stress.

All tests addressed are uniaxial, isothermal, experiments conducted at $650^{\circ} \mathrm{C}$, thus implying that the multiaxial material constants are typically generalized from their uniaxial counterparts. This need for generalization is precisely why a consistent multiaxial theory, such as that developed here from a potential formulation, is imperative. The available data is quite extensive, wherein five tests within the reversible domain (i.e., one creep with recovery upon complete unloading, one relaxation and 3 rate dependent effective/instantaneous moduli ), and eleven experiments conducted within the irreversible domain (i.e., three relaxation, four conventional creep, one multi-step creep, and three tensile curves at different total strain rates) have been used for characterization; while two multiple-step relaxation, one cyclic and one plasticity/creep interaction tests were reserved for validating the predictive behavior of the model.

It is important to realize from the outset, that the resulting set of material parameters is non-unique (due to the nonlinear nature of the problem), however if a sufficient amount of "data content" 5 is provided to COMPARE then it is believed that the final obtained parameters should be relatively independent of the initial guess and bounds provided. It is beyond the scope and intent of this paper, however, to define what constitutes this sufficient data content. For the purpose of the present exercise, the actual characterization process for the multimechanism viscoplastic portion of the model was divided into three stages given the fact that the viscoelastic constants were known from previous work, see ARNOLD ET AL. [1997, 2000], that is: 1$)$ determine the flow and hardening parameters $\left(\mu, n, H_{b}, \beta_{b}\right.$ and $\left.\kappa_{b}\right)$ using the three tensile curves at different total strain rates, 2 ) determine the thermal recovery parameters $\left(R_{b}\right.$ and $\left.m_{b}\right)$ given the three relaxation, four conventional creep, and one multi-step creep tests

\footnotetext{
${ }^{5}$ Data content is meant to imply the variety of experimental data provided not just quantity. In other words, if one only provided tensile data to COMPARE one should not expect the model to be able to accurately predict both creep and relaxation response (since the time duration of these types of tests far exceeds that of a tensile test). Similarly, if one provided only creep or relaxation data to COMPARE, one should not expect the model to accurately represent the typically shorter time domain tensile behavior of the material.
} 
Table 1: Final Viscoelastic material constants for TIMETAL 21S at $650^{\circ} \mathrm{C}$

\begin{tabular}{|c|c|c|c|c|c|c|c|}
\hline Constants & Units & \multicolumn{6}{|c|}{ Value } \\
\hline \hline $\mathrm{E}_{s}$ & $\mathrm{GPa}$ & 21.753 & & & & & \\
\hline$\nu$ & - & 0.365 & & & & & \\
\hline \hline Mechanisms & & $a=1$ & $a=2$ & $a=3$ & $a=4$ & $a=5$ & $a=6$ \\
\hline \hline $\mathrm{M}_{(a)}$ & $\mathrm{GPa}$ & 41.37 & 6.895 & 21.120 & 6.523 & 3.965 & 3.434 \\
\hline$\rho_{(a)}$ & $\mathrm{sec}$ & 0.5 & 50.0 & 974. & 9693. & $14,460.0$ & $28,218.0$ \\
\hline
\end{tabular}

Table 2: Final Viscoplastic material constants for TIMETAL $21 \mathrm{~S}$ at $650^{\circ} C$ given the saturating hardening form, $h_{\text {new }}$

\begin{tabular}{|c|c|c|c|c|c|c|c|}
\hline Constants & Units & \multicolumn{6}{|c|}{ Characterized Value } \\
\hline$\kappa$ & $\mathrm{MPa}$ & 4.25 & \multicolumn{2}{|c|}{4.25} & \multicolumn{3}{|c|}{4.25} \\
\hline$n$ & - & 1.016 & \multicolumn{2}{|c|}{1.016} & \multicolumn{3}{|c|}{1.01646} \\
\hline$\mu$ & GPa-sec & $289,303.2$ & \multicolumn{2}{|c|}{$289,303.2$} & \multicolumn{3}{|c|}{$221,264.0$} \\
\hline Mechanisms & & $b=1$ & $b=1$ & $b=2$ & $b=1$ & $b=2$ & $b=3$ \\
\hline$\kappa_{b}$ & $\mathrm{MPa}$ & 104.8 & 98. & 34.5 & 57. & 56.9 & 43.5 \\
\hline$H_{b}$ & GPa & 598. & 12.1 & 8939.3 & $19,000.7$ & 3.11 & 1.42 \\
\hline$\beta_{b}$ & - & 5.72639 & 2.785 & 1.0 & 1. & 6.64696 & 4.50 \\
\hline$R_{b}$ & $1 / \mathrm{sec}$ & $6.868827 \mathrm{E}-02$ & $2.2996 \mathrm{E}-03$ & $9.8679 \mathrm{E}-01$ & 1.5114 & $1.5483 \mathrm{E}-07$ & $1.7506 \mathrm{E}-02$ \\
\hline$m_{b}$ & - & 2.38539 & 1. & 1. & 1. & 4.02489 & 7.21429 \\
\hline
\end{tabular}

while keeping the flow and hardening parameter estimates from step 1 fixed and 3) fit all eleven tests at one time to obtain final parameter estimates - this is accomplished by allowing all viscoplastic parameters to be active (using the material parameters found in steps 1 and 2 as initial starting values) but with tight upper and lower bounds around each parameter. COMPARE was utilized exclusively in all stages of the characterization process, with the resulting set of viscoplastic parameters being given in Table 2. These three steps were then repeated for the cases when two and three viscoplastic mechanisms were active as well, the resulting parameter sets are also included in Table 2. For specific details regarding the required input to COMPARE, see the COMPARE User's guide, version 2.0, SAlEeb ET AL. [2001].

In the case of multiple viscoplastic mechanisms, it is important to remember that the bounds are selected so as to keep each mechanism separate from the other (as each mechanism should possess a unique internal clock, or effective time domain). Note that the initial starting value and both upper and lower bounds on the viscoplastic parameters were selected based on experience and physical meaning. For example, the maximum internal stress achievable (the hardening threshold, $\kappa_{b}$ ), which will strongly influence when a tensile stress-strain response saturates (given $h_{s a t}$ ), is equal to $\sum_{b=1}^{N} \kappa_{b}$; where $\mathrm{N}$ is the total number of viscoplastic mechanisms. This 
value $\left.\left(\sum_{b=1}^{N} \kappa_{b}=\left(\sigma_{\text {sat }}-\sqrt{3} \kappa\right) / \sqrt{3}\right)\right)$ can be estimated directly from the available constant strainrate tensile (stress-strain curves) tests, provided they have saturated. For TIMETAL 21S it appears that this total amount is in the range of 13 to $32 \mathrm{ksi}$ for the different strain rates used in the experiments. Note, in the above definition, $\sigma_{s a t}$ is defined to be the measured maximum stress, at the fully developed plastic/saturation state. Consequently, for the case of two and three mechanisms, the initial starting values for $\kappa_{b}$ are taken to be equal, i.e., $\kappa_{\text {single }} / 3$ and then allowed to move from there. The lower bound for $\sqrt{3} \sum_{b=1}^{N} \kappa_{b}$ being equal to the highest creep stress examined. Also, one is reminded that the threshold parameter $\kappa$ (representing that value of stress below which only reversible (albeit time-dependent or time-independent) behavior is present, i.e., no measurable inelastic behavior occurs) delineates the reversible and irreversible regimes. This is the reason why below $\kappa$ only viscoelastic behavior need be considered; alternatively when the stress state exceeds $\kappa$ both viscoelastic and viscoplastic behavior are fully active. Finally, it is highly recommended that the values of the exponents (i.e., $n, \beta_{b}$, and $m_{b}$ ) be always taken greater than one so as to ensure proper behavior of the various derivatives. Values less than one can at times produce excellent correlations while at the same time provide peculiar response histories for other loading configurations.

\section{Results:}

\subsection{Comparison of Saturating and Nonsaturating Hardening Forms}

In this section, we will examine the tensile response of TIMETAL $21 \mathrm{~S}$ over three orders of magnitude in total strain rate (i.e., $\dot{\varepsilon}=8.33 \times 10^{-4}, 8.33 \times 10^{-5}, 8.33 \times 10^{-6}$ per second) as well as its cyclic response in order to demonstrate the difference between the previously widely used nonsaturating hardening form [see Arnold\&Saleeb [1994], Arnold Et. AL. [1994a,1995], and SAleEb ET AL [2001]] and the newly introduced saturating form. The single mechanism representation in Table 2 will only be considered so that a consistent comparison can be made to our previous work (see SALEEB ET AL [2001]). Figure 1, illustrates the experimental stress strain response histories (indicated by symbols) and the corresponding correlated response via the saturated form (solid lines) and the nonsaturating form (dashed lines, see SALEEB ET AL [2001]). If we confine ourselves to the early portion ( $<1 \%$ total strain) of the response curves, see Fig. 1a, it is clear that both forms do an adequate job of capturing the experimental data over the range of strain rates. This however, is only true because we purposely limited the strain range to be less than or equal to $1 \%$. If we enlarge the strain range to that of $6 \%$ one can immediately see the significant difference between the two hardening functional forms, the saturating form being the more accurate representation overall. Similarly, in the case of cyclic loading (see Fig. 2) the saturating form does a significantly better job of representing the real material behavior over the entire strain range. Finally, simulations of other classes of loading (creep, relaxation, etc.) will not be adversely affected by the utilization of this new saturating hardening functional form, as demonstrated in the next section. 
Table 3: The Viscoplastic material constants for the previous, see Saleeb et al [2001], nonsaturating hardening function, $h_{\text {nonsat }}\left(G^{(b)}\right)$

\begin{tabular}{|c|c|c|}
\hline Viscoplastic & Units & Value \\
\hline \hline$\kappa$ & $\mathrm{MPa}$ & 4.24732 \\
\hline$n$ & - & 0.961 \\
\hline$\mu$ & $\mathrm{MPa}-\mathrm{sec}$ & $4.19216 \mathrm{e} 08$ \\
\hline$\beta_{(1)}$ & - & 0.333 \\
\hline$H_{(1)}$ & $\mathrm{MPa}$ & 74341.89 \\
\hline$m_{(1)}$ & - & 0.374 \\
\hline$R_{(1)}$ & $1 / \mathrm{sec}$ & $0.983 \mathrm{e}-04$ \\
\hline
\end{tabular}
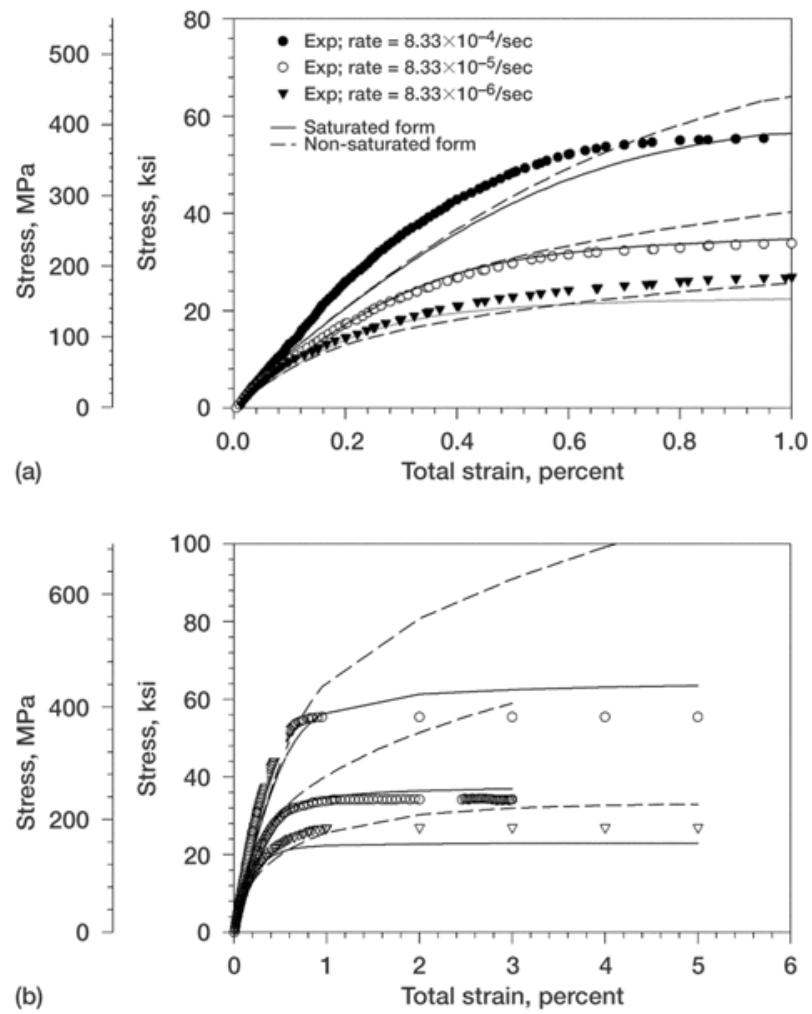

Figure 1: Comparison of specific hardening functions and their ability to represent the stressstrain behavior TIMETAL 21S over three different strain rates (i.e., 8.33E-04, 8.33E05 and $8.33 \mathrm{E}-06 / \mathrm{sec}$ ). a) strain range $0-1 \%$; b strain range $0-6 \%$. 


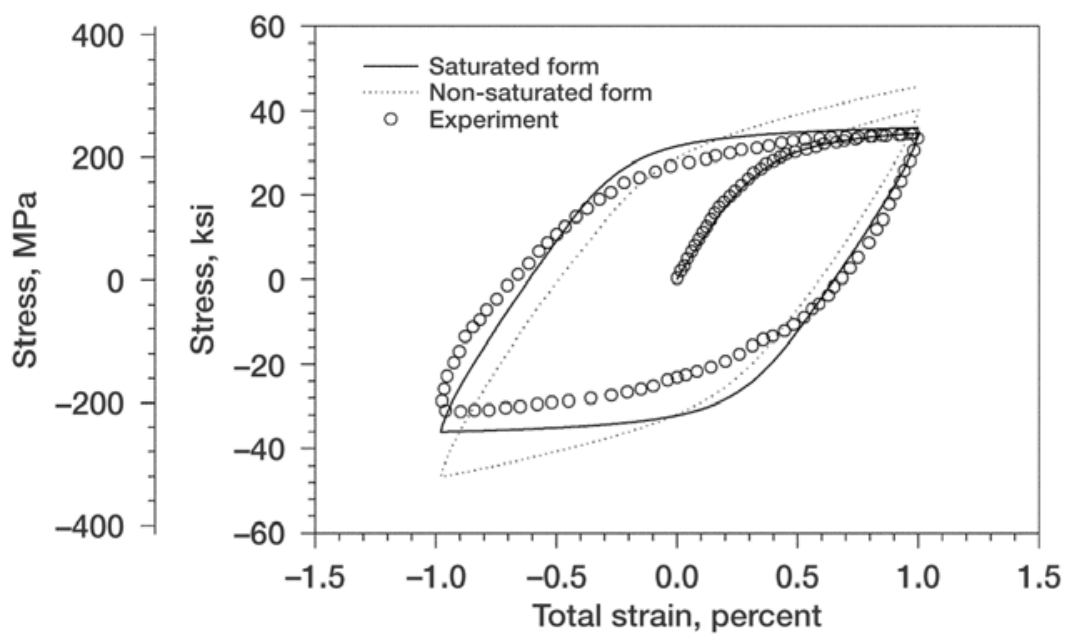

Figure 2: Comparsion of specific hardening forms under cyclic loading conditions.

\subsection{Correlation Capability of Single versus Multiple Mechanisms}

Here we examine the influence of including multiple viscoplastic mechanisms on the ability of the presented GVIPS class viscoplastic model to accurately simulate TIMETAL 21S. To date, only multiple mechanisms in the viscoelastic (reversible) portion of the model have been utilized, see ARNold ET AL. [1997]. In the following results, six viscoelastic mechanisms are included along with one, two or three viscoplastic mechanisms. In Figs. 3-6 the viscoelastoplastic correlations for, i) the tensile response of TIMETAL $21 \mathrm{~S}$ over three orders of magnitude in total strain rate (i.e., $\dot{\varepsilon}=8.33 \times 10^{-4}, 8.33 \times 10^{-5}, 8.33 \times 10^{-6} / \mathrm{sec}$ ), ii) relaxation responses given three initial strain levels (i.e., $\varepsilon=139,400$ and $602 \mu \varepsilon$ loading, respectively, to the following "peak" stress levels, $\sigma=103,238$, and $345 \mathrm{MPa}$, all produced given a total strain rate of $\dot{\varepsilon}=5.0 \times 10^{-4} / \mathrm{sec}$ ), and iii) short term creep at four stress levels (i.e., $\sigma=14,72,110$, and 128 $\mathrm{MPa}$ ) and one multiple step creep test are shown, respectively. In the graphs, the symbols denote experimental data and the lines the model correlations, with the solid line denoting a single mechanism; dotted line - two, and the dashed line - three viscoplastic mechanisms. In the authors' opinions, these correlations (irrespective of the number of mechanisms utilized) are qualitatively very good given the very wide variety of loading conditions examined. Overall the maximum quantitative correlation errors are between $1 \%$ to $50 \%$ depending upon the load history imposed in a given test. This is well within typical experimental scatter, particularly when dealing with creep measurements which can show upwards of $200-300 \%$ variation.

Examining the tensile responses, shown in Fig. 3, it is clear that the model with a single (or multiple) irreversible dissipative mechanism is able to qualitatively capture the ratedependency of the material. Note how only the $8.33 \times 10^{-5} / \mathrm{sec}$ strain rate test is quantitatively accurate with the lower and higher strain rates being quantitatively in greater error. This is expected, since the single irreversible dissipative mechanism (although stress dependent) must compromise over the entire time spectrum of all the experiments being correlated. Clearly, 
given the current data set, this mechanism will be biased toward the longer time domain as eight of the eleven experiments are long term. When three mechanisms are considered the model appears to be better able to accurately capture (both qualitatively and quantitatively) the actual strain rate dependency of the material.

Qualitatively the relaxation behavior exhibited by the material, shown in Fig. 4, is very well captured by the model again irrespective of the number of viscoplastic mechanism used. This is particularly true with regard to the primary relaxation feature, that is, the initially high stress reduction rate and overall large stress-drops at all stress levels. Note that the three starting relaxation stress levels in Fig. 4 represent stress levels ${ }^{6}$ that are: i) below the apparent yield, ii) within the knee (e.g. 115 to $330 \mathrm{MPa}$ ), and iii) near the ultimate stress level of the material (approximately $345 \mathrm{MPa}$ ), see Figs. 3 and inserts of Fig. 4. In order to accurately capture (quantitatively) the entire relaxation history it appears to require at least two mechanisms be incorporated within the model (three being slightly better).

Similarly, when examining the creep response correlations given in Fig. 5, the model again, irrespective of the number of viscoplastic mechanism used, is able to capture qualitatively the stress dependence over the entire stress range. However, clearly, incorporating additional viscoplastic mechanisms (three being overall the most accurate) enhances the present model's ability to accurately (quantitatively) simulate both short term and longer term creep behavior. Note, how the secondary creep rate is significantly more accurate when three mechanisms are utilized. Figure 6 shows the correlation of the multiple step creep test, where the resulting inelastic strain versus time (at constant stress) is shown. Here the simulation using multiple viscoplastic mechanisms (two - dotted and three - dashed lines), as compared with the experimental data (denoted by the symbols), is seen to accurately represent the first two steps but under-predict the accumulated inelastic strain of the third step. This is not surprising, however, given the fact that this test was performed under constant load and not constant stress as was the simulation. As a consequence of the constant load controlled creep test, the experimental results have both additional geometric effects and possibly creep damage (thereby leading to tertiary creep) included in them. Neither of these factors have been included in the numerical simulation. The influence of these factors is particularly evident during the third step, where the inelastic strain rate during the experiment is significantly greater than that simulated (cf. $\sigma=128 \mathrm{MPa}$ in Fig. 5d).

It is important to note that if one were to focus on only one type of loading condition, superior correlations could of course be achieved for that class of loading. However, predictions of other classes of loading may severely suffer. For example, during a separate characterization process involving only the three tensile tests, excellent correlation was obtained, but at the expense of poor creep and relaxation behavior for a given set of material parameters. Conversely, when the model was calibrated for creep responses, poorer tensile and relaxation behavior were similarly predicted. Thus when judging the "goodness" of a given model one must keep in mind the appropriate required "data content" for accurately capturing the full range of material behavior of interest. Similarly when comparing the current correlation with that done previously (see SALEEB ET AL. [2001]) it should be remembered that here all exponents were constrained to be always greater than or equal to one, even though superior correlations in some cases could be obtained if one used exponents (in particular $\beta$ ) that are less than one. This latter condition

\footnotetext{
${ }^{6}$ Given a $5.0 \times 10^{-4} /$ sec total strain rate ramp-up history, see Fig. 4.
} 

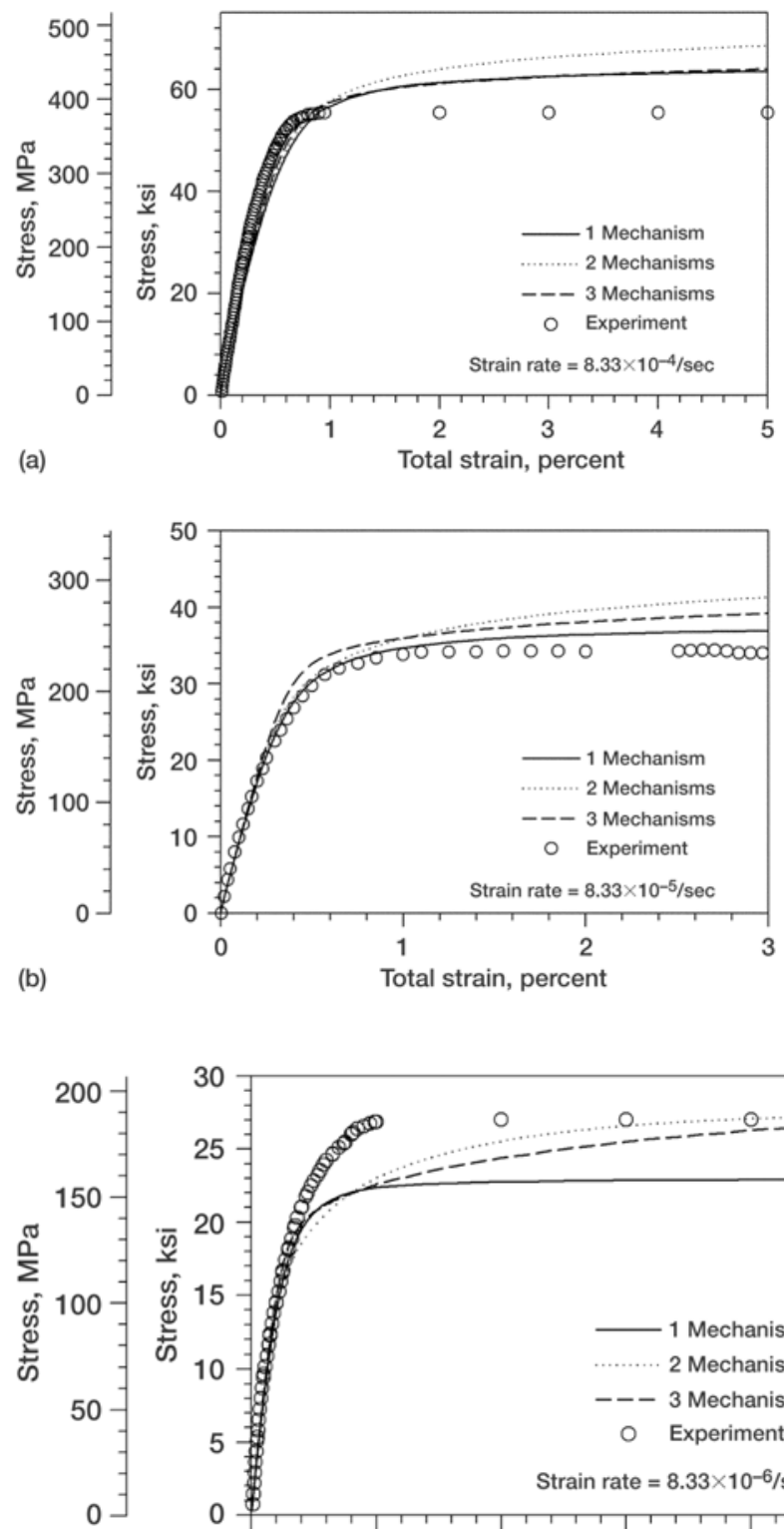

(c)

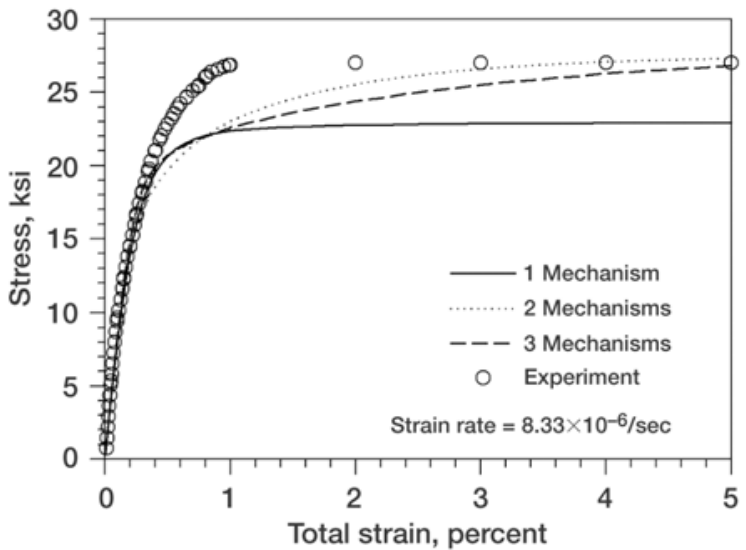

Figure 3: Illustrates the correlation capability of present model when one, two or three viscoplastic mechanisms are employed given constant strain rate, tensile histories; i.e., a) $8.33 \mathrm{E}-04, \mathrm{~b}) 8.33 \mathrm{E}-05$ and c) $8.333 \mathrm{E}-06 \mathrm{~m} / \mathrm{m} / \mathrm{sec}$ 


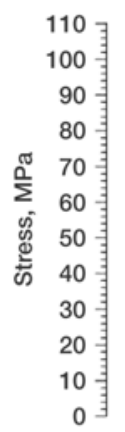

(a)

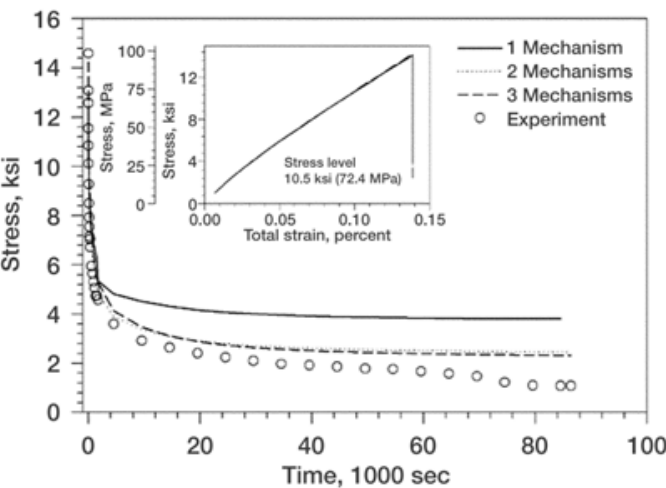

(b)

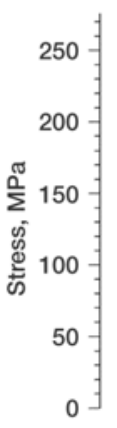

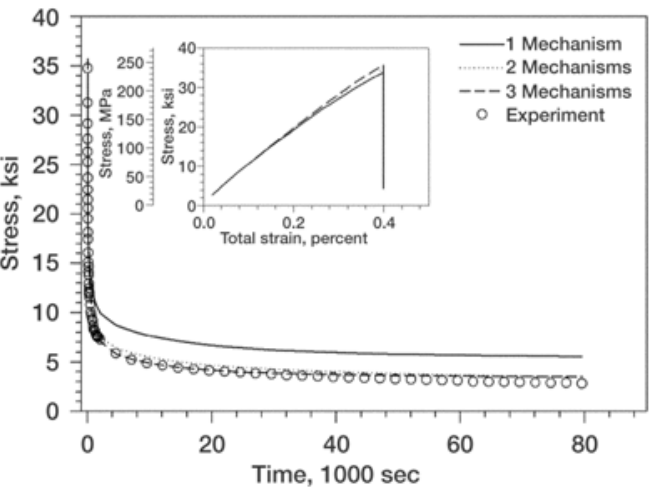

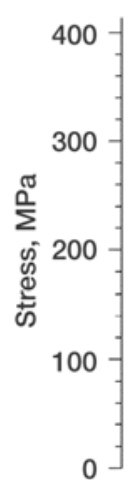

(c)

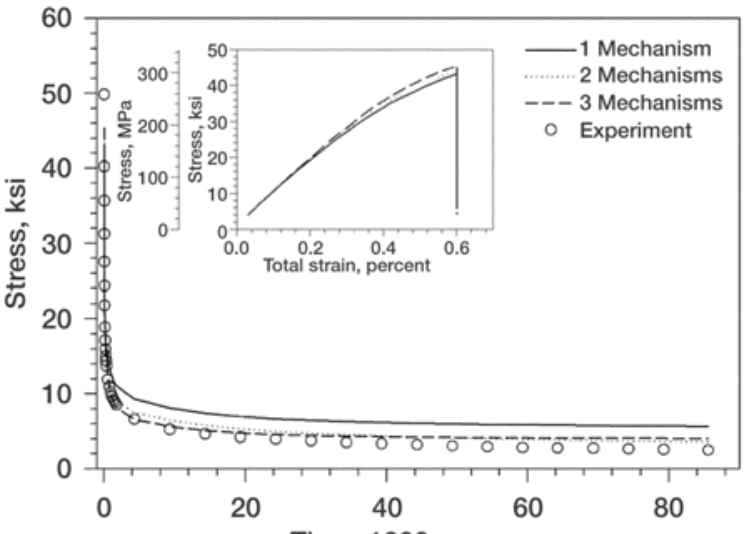

Figure 4: Illustrates the correlation capability of the present model when one, two or three viscoplastic mechanisms are employed given relaxation histories. Peak stress levels are: a) $103 \mathrm{MPa}$, b) $238 \mathrm{MPa}$ and c) $345 \mathrm{MPa}$ 

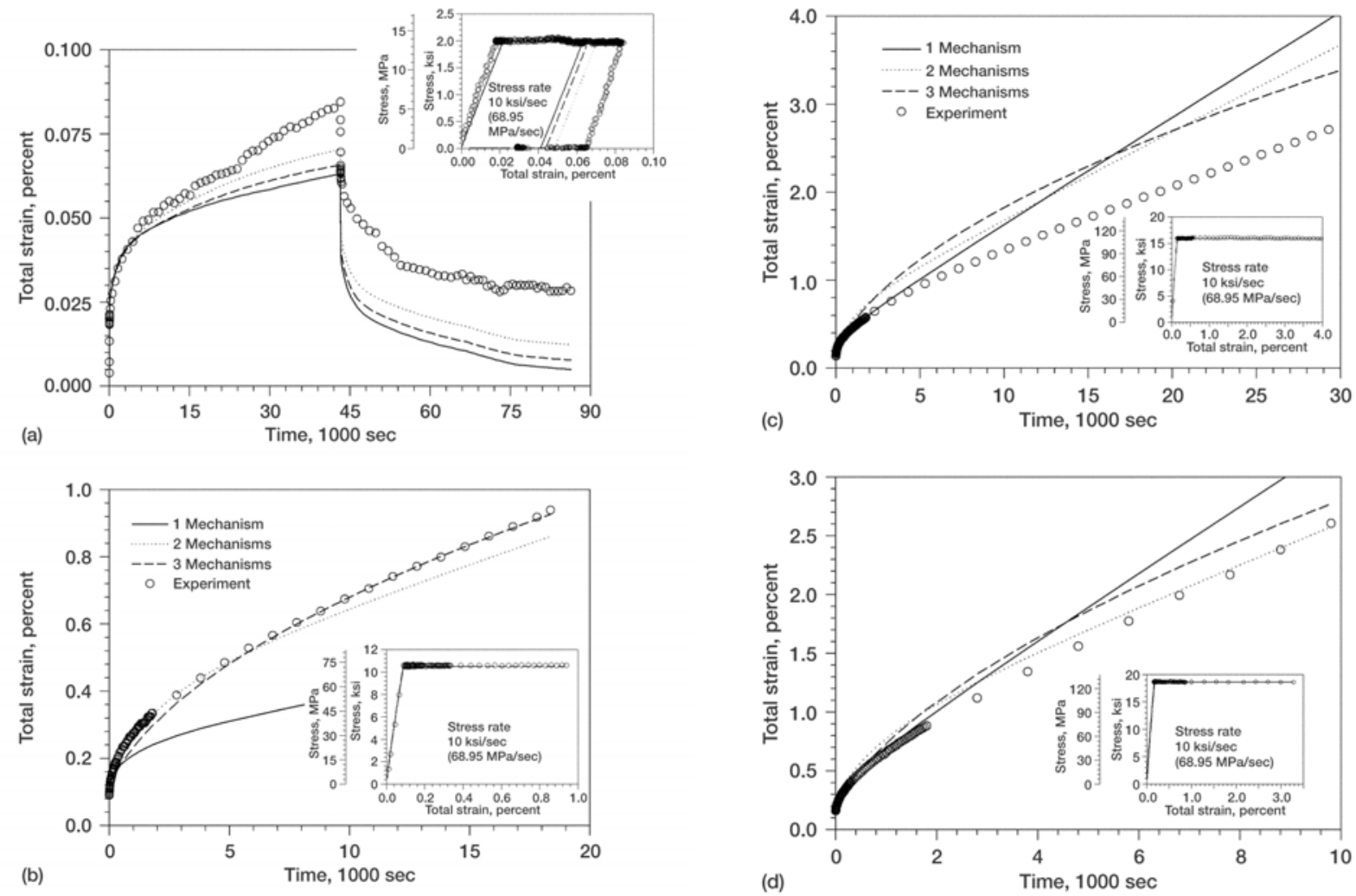

Figure 5: Illustrates the correlation capability of the present model when one, two or three viscoplastic mechanisms are employed in the context of creep (constant stress) loading. The applied stress is: a) 14, b) 72 , c) 110 and d) $128 \mathrm{MPa}$ 


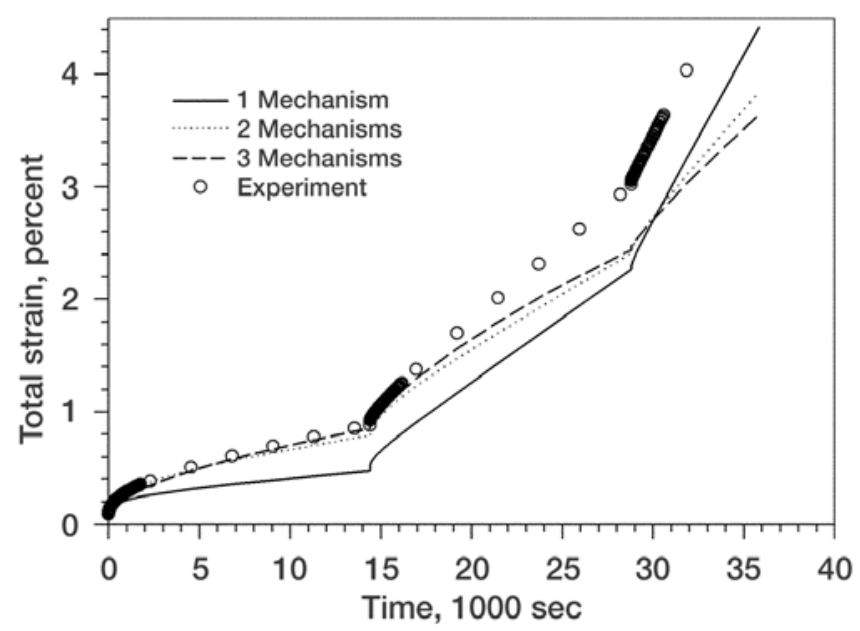

Figure 6: Illustrates the correlation capability of the present model when one, two and three mechanism are used, given a multistep load history, i.e., 72,110 and $128 \mathrm{MPa}$ steps.

was implemented since some anomalies in certain response histories were observed due to higher order derivatives of the hardening and recovery functions employed in the implicit integration algorithm. This derivative can become problematic when exponent values less than one are used.

\subsubsection{Back Stress Partitioning within Irreversible Domain}

To gain insight into and help explain the differences between the use of a single irreversible mechanism and that of multiple mechanisms, regarding the evolution of the effective stress $\left(\boldsymbol{\sigma}_{i j}-\boldsymbol{\alpha}_{i j}\right)$ which drives the inelastic response of the material, we have graphed the uniaxial external stress $(\boldsymbol{\sigma})$, individual internal (back) stresses, $\boldsymbol{\alpha}^{(b)}$, for each mechanism, as well as the sum of the back stresses, $\alpha$, for each class of loading examined, that is, tensile, creep, relaxation and cyclic; see Figs. 7 through 10.

Figure 7 illustrates the stress versus time response corresponding to the tensile response (given a total strain rate of $\dot{\varepsilon}=8.33 \times 10^{-5} / \mathrm{sec}$ ) shown in Fig. 3b. It is clearly evident from Fig. 7 that as the externally applied stress increases so too does the internal (or non-equilibrium) stress, albeit at a slower rate, until the maximum value of the back stress is reached. This maximum (uniaxial) value being related to the obtained (estimated) shear threshold value $\kappa_{b}$, i.e., $\sqrt{3} \kappa_{b}$. The determined shear threshold value for this material is $104.7 \mathrm{MPa}$ in the case of the single viscoplastic mechanism and $158 \mathrm{MPa}$ (for the sum) in the case of three viscoplastic mechanisms (see Table 2). The fact that the non-equilibrium stress (internal stress)does not go to zero after an "infinite" amount of time, but rather reaches a constant value is a distinguishing feature between the reversible and irreversible time dependent modeling.

Figure $7 \mathrm{~b}$ demonstrates clearly the existence of different internal viscoplastic clocks, since the rate of growth of the first mechanism (depicted by the solid line) is relatively short as compared to mechanisms two and three (as well as the case of a single mechanism, shown in 

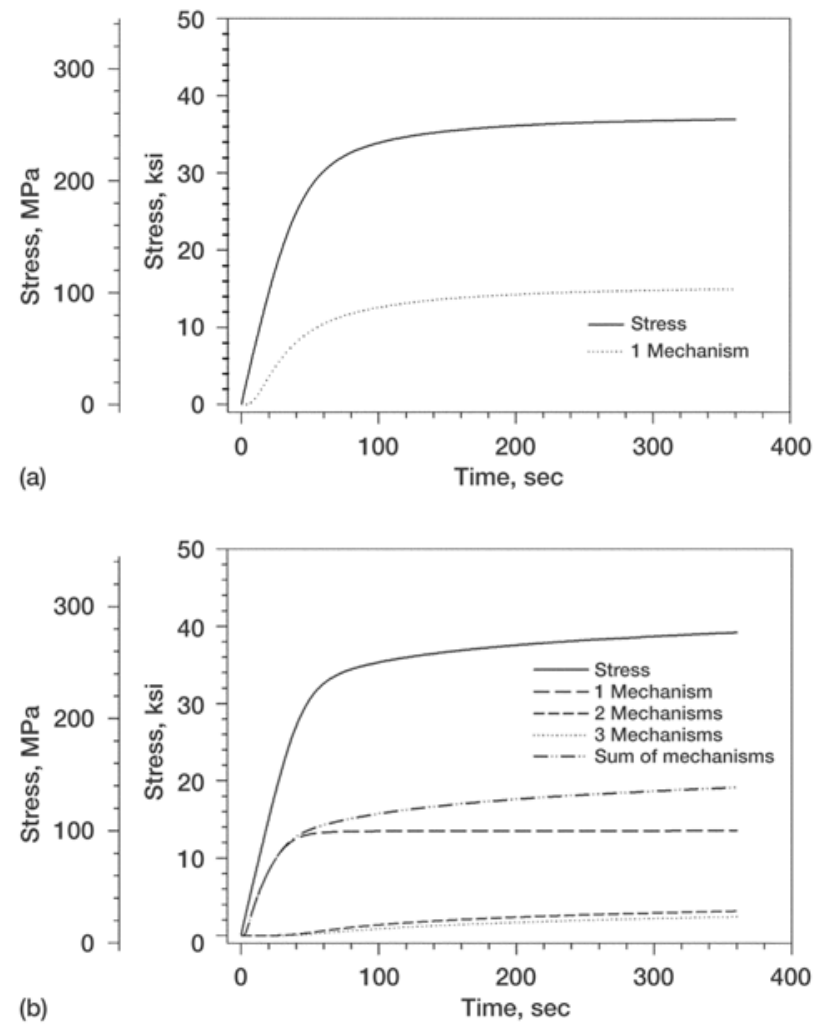

Figure 7: Illustrates the evolution in time of both the external and internal (for each mechanism) stress fields during the tensile response shown in Fig. 3b. 
Fig 7a). Specifically, in the single mechanism case the internal stress saturates after approximately 300 seconds whereas the first mechanism in the three mechanism model saturates after approximately 100 secs. Further, one can see that the maximum value of internal stress reached by mechanism-one (see Fig. 7b) is very near (i.e., $93.4 \mathrm{MPa}$ ) its threshold value of 98.7 (or 57.0 in shear) $\mathrm{MPa}$. This is in sharp contrast to the single mechanism case wherein the internal stress essentially saturates at $103 \mathrm{MPa}$ which is substantially below its threshold value of 189.6 (or 104.7 in shear) MPa. The reason for the "apparent" premature saturation stems from the larger hardening exponent, $\beta=5.726$, used in the case of a single mechanism whereas $\beta=1$ was employed for the first mechanism in the three mechanism model. The other two constant strain rate $\left(\dot{\varepsilon}=8.33 \times 10^{-4}\right.$ and $\left.8.33 \times 10^{-6} / \mathrm{sec}\right)$ tensile tests exhibit qualitatively the same (stress-time) behavior with only the actual magnitude of both internal and external stress being different. The ordering of the final resulting equilibrium (effective) stress (i.e., $(\boldsymbol{\sigma}-\boldsymbol{\alpha})$ ) at saturation of the externally applied stress (for all three tensile tests) is consistent with the applied total strain rate, i.e., the faster the rate the higher the effective stress. This should not be surprising, since from eqn. 25 it is immediately apparent that after sufficient time has elapsed (i.e., $\dot{\mathbf{q}}=0$ ) and the change in external stress has stopped (i.e., $\dot{\boldsymbol{\sigma}}=\mathbf{0}$, the tensile curve has flattened), the inelastic strain rate $\left(\dot{\varepsilon}^{v p}\right)$ is equal to the applied total strain rate $(\dot{\varepsilon})$ and is consequently proportional to the equilibrium (effective) stress.

Figure 8 illustrates the stress versus time response corresponding to the creep response (given an applied stress of $\sigma=72.0 \mathrm{MPa}$ ) shown in Fig. 5b. The single mechanism representation shown in Fig 8a is very similar to the tensile case shown in Fig. 7a, in that initially one has a rapid increase in internal stress followed by a saturation of the internal stress. The nonlinear nature of the response is apparent when one compares the "steady-state" response of Figs. $7 \mathrm{a}$ and $8 \mathrm{a}$, that is $\left(\boldsymbol{\sigma}-\boldsymbol{\alpha} \approx 151.7 \mathrm{MPa}, \dot{\varepsilon}=8.33 \times 10^{-5} / \mathrm{sec}\right)$ and $(\boldsymbol{\sigma}-\boldsymbol{\alpha} \approx 17.9 \mathrm{MPa}$, $\dot{\varepsilon}=1.8 \times 10^{-7} / \mathrm{sec}$ ). The behavior of the multimechanism model (see Fig. $8 \mathrm{~b}$ ) is in sharp contrast, however, to that of the single mechanism model; in that, although the overall internal stress does indeed still rise in time (albeit at a much slower rate) the first mechanism actually decreases (or relaxes). Also, examining the time corresponding to "constant" internal stress (i.e. steady-state), it is clear from Fig. 8 that the single mechanism arrives at this condition in approximately 1500 secounds whereas the three mechanism model requires more than 15000 secs, thus indicating once again a significant difference in the individual internal irreversible material time clocks. Figure 5b clearly illustrates that the three mechanism response is significantly more accurate than the single mechanism with the "steady-state" strain rate of approximately $3.4 \times 10^{-07} /$ sec being very well represented given an effective stress of approximately 20.6 MPa.

Similar behavior to that described above is observed for relaxation and cyclic loading cases, see Figs. 9 and 10. It is interesting to note that in the case of relaxation (Fig. 9), the relaxation of the internal stress follows that of the external stress almost exactly, especially for the single mechanism representation, except early on where the internal stress reached a maximum value of $42.7 \mathrm{MPa}$ after 593 seconds. Alternatively, in the three mechanism case the maximum internal stress in the first mechanism (which is the dominate mechanism) is approximately 51.0 $\mathrm{MPa}$ after 3.9 seconds and subsequently relaxes to $4.5 \mathrm{MPa}$ after 80,000 secs (the sum being 8.4 $\mathrm{MPa}$ ). In the case of cyclic loading (Fig. 10) it again is clear that the single mechanism and first mechanism of the three mechanism model have very similar internal clocks as the evolution of internal stress is almost identical over the entire load history. Furthermore, it is clear that the first mechanism dominates this load case. 

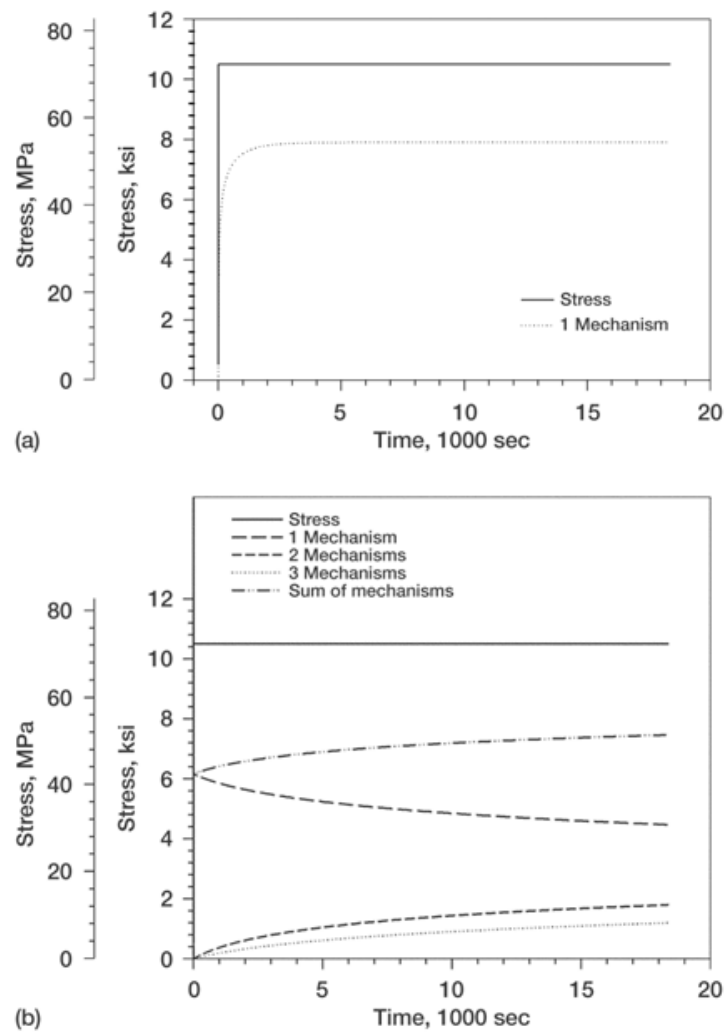

Figure 8: Illustrates the evolution in time of both the external and internal (for each mechanism) stress fields during the creep response shown in Fig. 5b. 

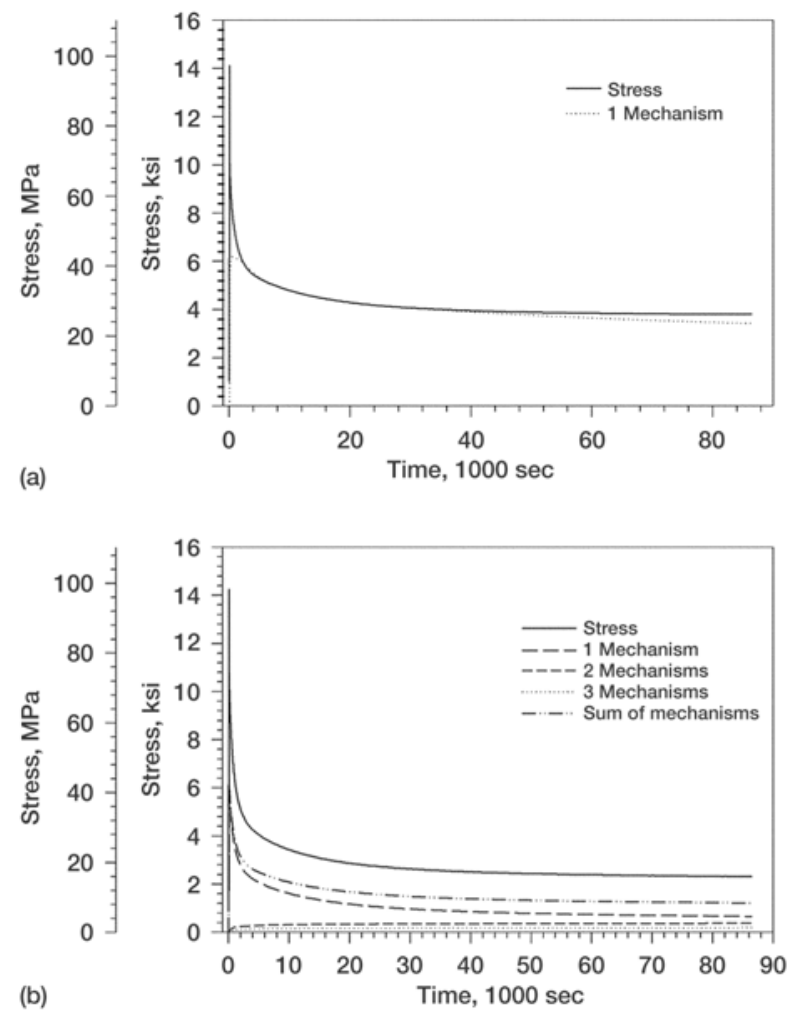

Figure 9: Illustrates the evolution in time of both the external and internal (for each mechanism) stress fields during the relaxation response shown in Fig. 4a. 


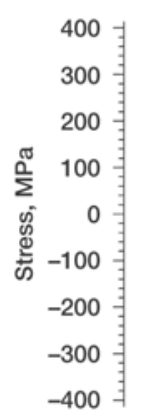

(a)

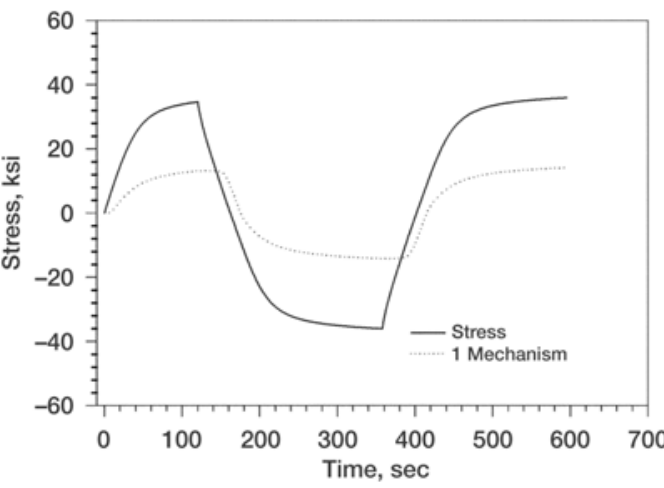

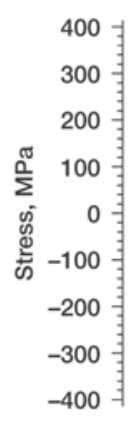

(b)

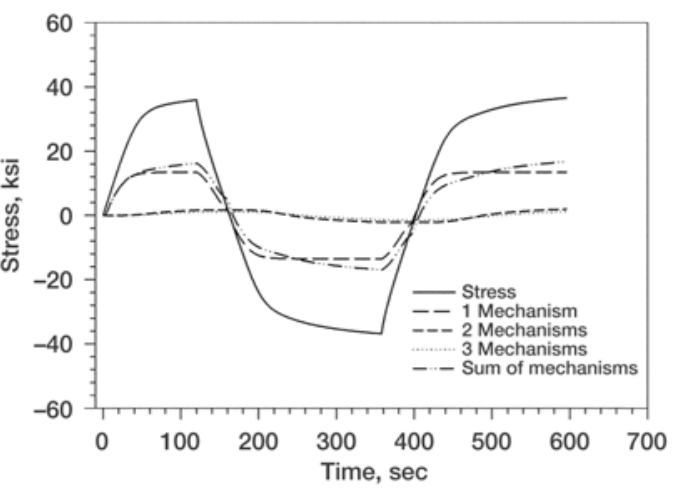

Figure 10: Illustrates the evolution in time of both the external and internal (for each mechanism) stress fields during the cyclic response of Figs. 2 and 11. 


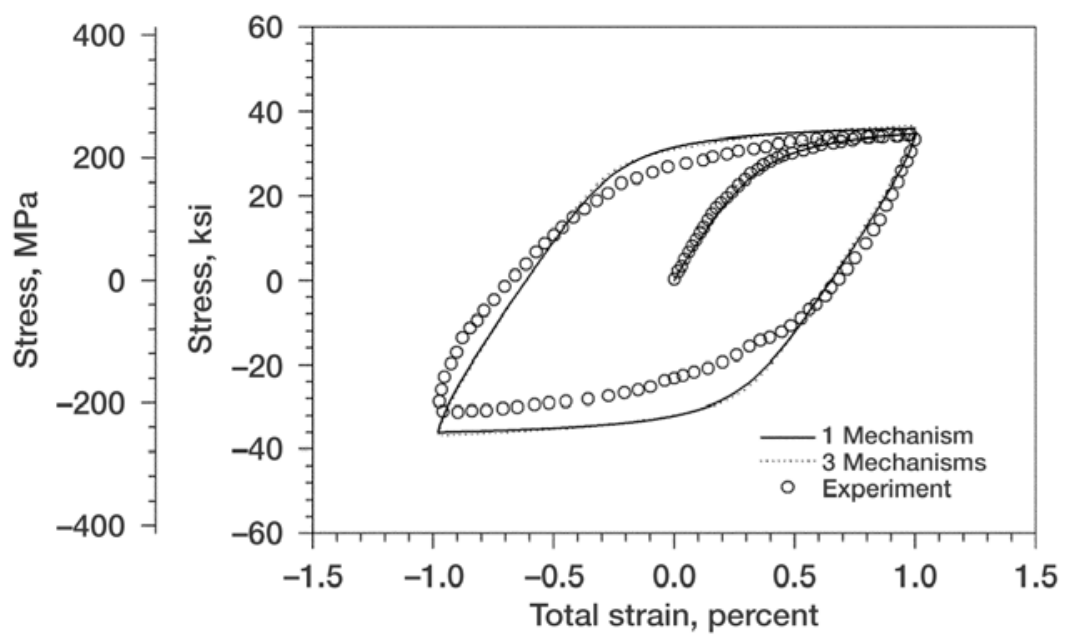

Figure 11: Illustrates the predictive capability of a single and three viscoplastic mechanism model subjected to a cyclic history, given a constant strain rate of $8.33 \times 10^{-5} / \mathrm{sec}$.

\subsection{Predictive Ability of Single versus Multiple Mechanisms}

The present viscoelastoplastic multimechanism GVIPS model, characterized to represent the behavior of TIMETAL $21 \mathrm{~S}$ at $650^{\circ} \mathrm{C}$, is now exercised and its predictive capability assessed relative to the number of viscoplastic mechanisms employed. The assessment begins by considering a cyclic test, followed by multi-step relaxation tests, creep reversal or recovery tests and finally a plasticity/creep interaction test.

Figure 11 illustrates the cyclic behavior under strain control given a total strain rate of $8.33 \times 10^{-5} / \mathrm{sec}$, wherein the symbols represent the experimental results and the solid (single mechanism) and dotted (three mechanism) line the GVIPS prediction. Here we see that the agreement is very good with less than a $0.5 \%$ over-prediction of the maximum stress at +0.01 and $20 \%$ at -0.01 strain. This is in sharp contrast to our previous (see SALEEB ET AL [2001]) predictive results employing the non-saturating hardening form, see Fig. 2, where a $17 \%$ overprediction of the maximum stress at +0.01 and $45 \%$ at -0.01 strain was obtained. Clearly, when using the current saturating hardening form, either, a single or multiple mechanism formulation provides the same level of accuracy.

Multistep relaxation tests were performed to document the influence of prior history on the time-dependent response of TIMETAL $21 \mathrm{~S}$ and validate the predictive capability of the present model(see SALEEB ET AL [2001]). The two-step load sequence was comprised of a 345 MPa stress level, relaxed for 24 hours followed by an initial $103 \mathrm{MPa}$ stress level relaxed for approximately one hour. The experimental stress versus time response and GVIPS simulations given multiple mechanisms are shown in Fig. 12. The first step of this two step relaxation test was used for correlation purposes (see Fig. 4c), with the second step shown in Fig. 12 being a measure of the predictive capability of the model. Clearly, in this case the two mechanism characterization is more accurate than the three mechanism case. Similarly, in the case of the three step relaxation load sequence (shown in Fig. 13, wherein step 1 began at a stress 


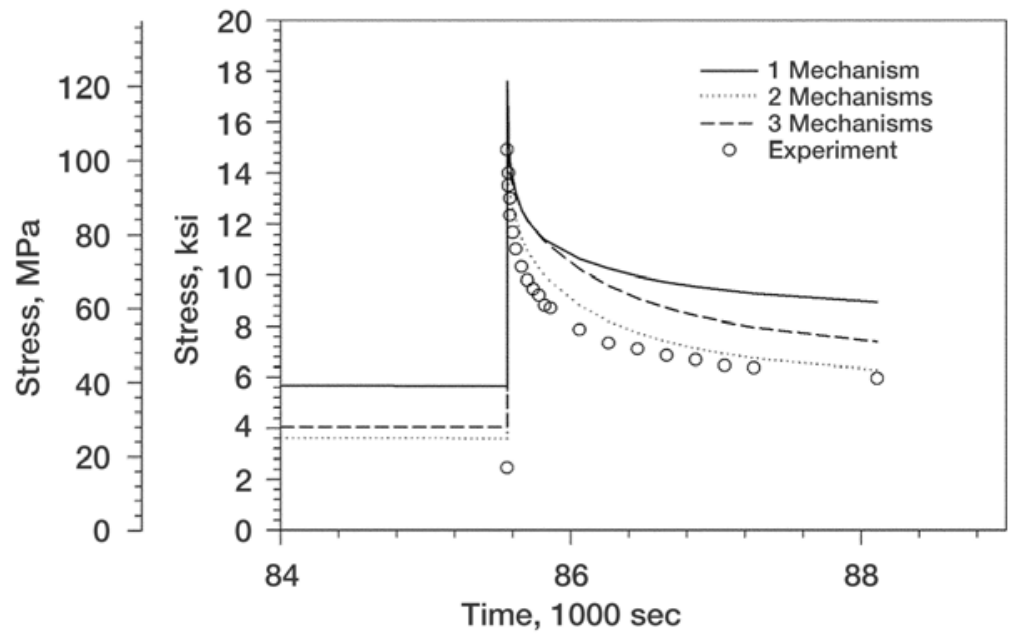

Figure 12: Illustrates the predictive capability of a single viscoplastic and multimechanism models subjected to a two-step relaxation history. Here only the response corresponding to the second step is shown. The starting stress level being $103 \mathrm{MPa}$.

level of $103 \mathrm{MPa}$, step 2 at $238 \mathrm{MPa}$ and step 3 once again at $103 \mathrm{MPa}$ with each constant total strain hold period lasting approximately 24 hours) the two mechanism model appears once again to slightly out perform the three mechanism model. Obviously, these results demonstrate that a single mechanism viscoplastic GVIPS model is not capable of accurately capturing the influence of multistep load histories when compared to multimechanism representations.

Lastly, a classic plasticity-creep interaction experiment, which illustrates the need for unified viscoplastic models, was performed and simulated. In this type of experiment the material is subjected to an overload prior to performing a creep test at a lower stress value, see Fig. 14. Typically, as is found here, the initial creep response following the overload (denoted by the open circle symbols) is significantly reduced when compared to that produced from a pure creep test (denoted by the open triangles) at this lower stress amplitude, see Fig. 14b. Once again comparing the numerical simulations (for the one, two and three mechanism GVIPS models) to that of the experimental results one observes very good overall agreement for the multimechanism simulations; whereas, for the single mechanism case approximately a $50 \%$ error is incurred. For this type of loading history, it appears that the three mechanism model slightly out performs the two mechanism model.

\section{Conclusions}

Hereditary behavior encompasses such important features as nonlinearity, loading-rate sensitivity, creep, relaxation, creep-plasticity interaction effects, as well as memory effects for both the short-term (transient) and long-term (steady states of limit equilibrium) material responses. To date a general, potential-based, viscoelastoplastic multimechanism deformation model has been formulated and implemented. In this study, two specific material hardening functions 


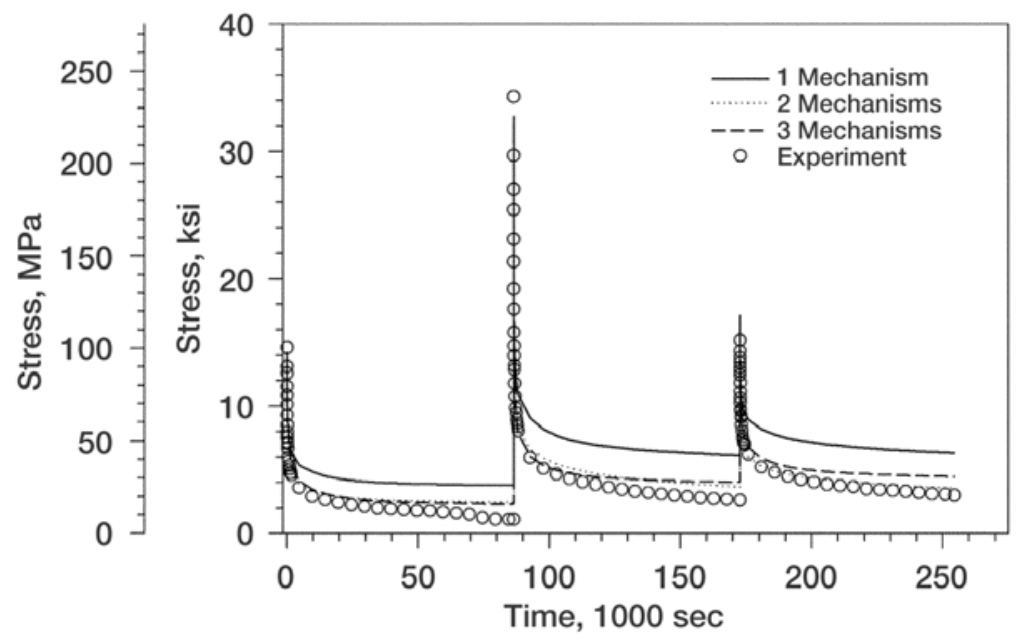

Figure 13: Illustrates the predictive capability of a single viscoplastic and multimechanism models subjected to a three-step relaxation history. The starting stress levels for each step being, 103, 238 and $103 \mathrm{MPa}$, respectively.

have been evaluated as well as the influence of employing multiple viscoplastic mechanisms as opposed to only a single mechanism. Material characterization of a titanium based alloy, TIMETAL 21S, was accomplished using the recently developed COMPARE software, a fullyautomated procedure for material parameter estimation, and a fairly comprehensive set of test data under different loading conditions. In addition to these correlation tests, several other experimental results were reserved to assess the predictive capability of the model.

The overall performance of the multimechanism model was found to be very good, clearly demonstrating the great potential, effectiveness, as well as practical utility of the proposed model. In particular, the utilization of a material hardening function $(h(G))$ that saturates when the internal stress reaches a threshold value was found to be superior to the previously used non-saturating form when relatively rapid rates of loading (i.e. on the order of seconds and minutes as opposed to miniutes and hours) are imposed, as typical in strain controlled tensile tests. Furthermore, it was clearly demonstrated in this study that the introduction of this saturating hardening functional form also demands the use of more than a single viscoplastic sub-mechanism if one desires to accurately represent both relaxation and creep behavior as well as tensile behavior. Finally, the existence of multiple internal clocks which account for the wide range in observed response history is illustrated by plotting the evolution of internal (non-equilibrium) stress within the multimechanism model. The coupled nature of the model is clearly seen in the external and internal stress plots (see Figs. 7 through 10) wherein for example one internal stress may rise/fall while the other(s) fall/rise. Accurate results, both qualitatively and quantitatively, over the entire load-time spectrum, can be achieved with the use of additional sub-mechanisms within the irreversible domain. This conclusion is consistent with our previous findings within the reversible domain (the need for 6 viscoelastic mechanisms, to capture both time dependency and rate-sensitivity) wherein multiple mechanism were required to accurately represent the deformation behavior over a wide time spectrum, see Arnold ET. AL. [1997]. 

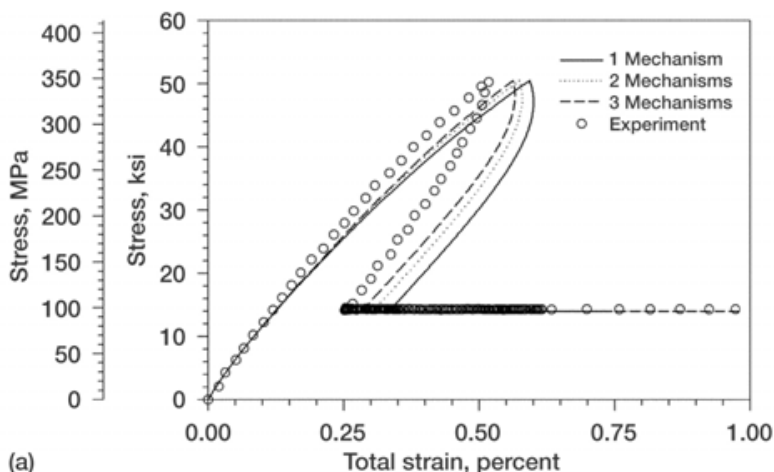

(a)

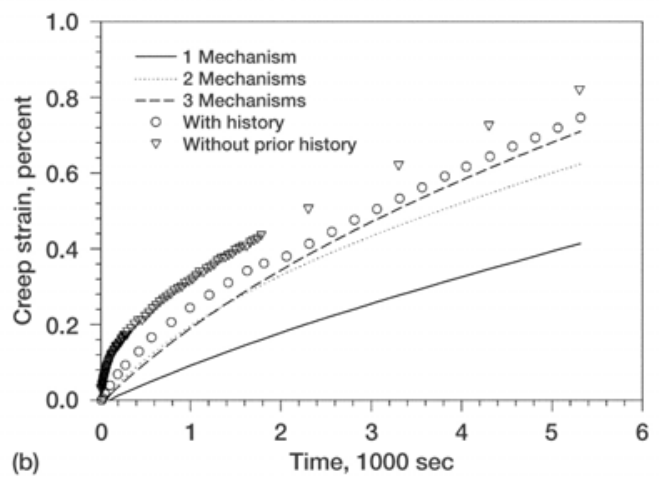

Figure 14: Illustrates the predictive capability of a single and multimechanism model subjected to a plasticity/creep interaction history: a) stress versus total strain response and b) creep strain versus time response. 


\section{References}

[1967] Coleman., B.D. And Gurtin, M.E.: "Thermodynamics with Internal "State Variables", J. Chem. Phys., Vol. 47, No.2, pp. 597-613.

[1973] Lubliner, J.: "On the Structure of Rate Equations of Materials with Internal Variables," Acta Mechanica, Vol. 17, pp. 109-119.

[1976] Hart, E.W.: "Constitutive Relations for Nonelastic Deformation of Metals", J. Eng. Mater. Technol., Vol. 98, pp. 193-202.

[1980] Kumar, V., Morjaria, M. and Mukherjee, S.: Numerical Integration of Some Stiff Constitutive Models of Inelastic Deformation", J. Eng. Mater. Technol., Vol. 102, pp.92-96.

[1982] Hirth, J.P. And Lothe, J.; Theory of Dislocations, Wiley, N.Y..

[1985] Hui, C.Y. : "A Uniformly Valid Asymptotic Solution of Hart's Equations for Constant Inelastic Extensional Strain Rate", Int. J. Solids and Struct., Vol. 21, pp. 411-421.

[1987] Robinson, D.N. : "A Unified Creep-Plasticity Model for Structural Metals at High Temperatures", ORNL TM-5969.

[1989] Chaboche, J.L.: "Constitutive Equations for Cyclic Viscoplasticity", Int. J. Plasticity, Vol. 5, pp. 247-302.

[1990] Lemaitre, J.; and Chaboche, J.L.: Mechanics of Solid Materials, Cambridge University. Press, New York.

[1993] Ashbaugh, N.E., And Khobaib, M.: Unpublished Data, University of Dayton Research Institute, Dayton, Ohio.

[1993] Freed, A.D. And Walker, K.P.: "Viscoplasticity with Creep and Plasticity Bounds, Int. J. Plasticity, Vol. 9, pp. 213-242.

[1993] Saleeb, A.F. And Wilt, T.E.: "Analysis of the Anisotropic Viscoplastic-Damage Response of Composite Laminates-Continuum Basis and Computational Algorithms," Int. Jnl. Num. Meth. Engng., Vol. 36, pp. 1629-1660.

[1994] Arnold, S.M.; And Saleeb, A.F.: "On the Thermodynamic Framework of Generalized Coupled Thermoelastic Viscoplastic - Damage Modeling", Int. J. Plasticity, Vol. 10, No. 3, pp. 263-278.

[1994a] Arnold, S. M., Saleeb, A.F., And Castelli M.G.: "A Fully Associative, Nonlinear Kinematic, Unified Viscoplastic Model for Titanium-based Matrices", Life Prediction Methodology for Titanium Matrix Composite, ASTM STP 1253, W.S. Johnson, J.M. Larsen, and B.N. Cox, Eds. American Society for Testing and Materials, Philadelphia, pp. 231-256, or NASA TM-106609. 
[1994b] Arnold, S.M.; Saleeb, A.F., And Castelli, M.G.: A Fully Associative,Nonisothermal, Nonlinear Kinematic, Unified Viscoplastic Model for Titanium Based Matrices, Thermomechanical Fatigue Behavior of Materials: Second Volume, ASTM STP 1263, M.J. Verrilli and M.G. Castelli, Eds., Philadelphia,or NASA TM106926.

[1995] Arnold, S.M., Saleeb, A.F, And Wilt, T.E.: " A Modeling Investigation of Thermal and Strain Induced Recovery and Nonlinear Hardening in Potential Based Viscoplasticity," J. Engng. Mater. Tech. ASME, Vol. 117, pp. 157-167.

[1996] Chaboche, J.L. and Cailletaud, G.: "Integration Methods for Complex Plastic Constitutive Equations", Comput. Meth. Appl. Mech. Eng., Vol. 133, pp. 125-155.

[1996] Krempl, E. : "A Small Strain Viscoplasticity Theory Based on Overstress", In: Unified Constitutive Laws of Plastic Deformation, Krausz, A and Krausz, K. (Eds.), Academic, San Diego, pp. 281-318.

[1996] Krausz, A.S. and Krausz, K.(eds.): "Unified Constitutive Laws of Plastic Deformation", Academic Press, New York.

[1997] Saleeb, A.F. And Arnold, S.M.: "A General Reversible Hereditary Constitutive Model: Part I: Theoretical Developments", NASA TM-107493; also see Jnl. Eng. Mat. Tech., Vol. 123, pp. 51-64, 2001.

[1997] Arnold, S.M.,Saleeb, A.F. And Castelli, M.G.: "A General Reversible Hereditary Constitutive Model: Part II: Application to A Titanium Alloy", NASA TM 107494; also see Jnl. Eng. Mat. Tech., Vol. 123, pp. 65-73, 2001.

[1998] Saleeb,A.F., Wilt, T.E., And Li, W.: "An Implicit Integration Scheme for Generalized Viscoplasticity with Dynamic Recovery", Comp. Mech., Vol. 21, No. 6, pp. 429-440.

[1998] Saleeb, A.F., Gendy, A.S., Wilt, T.E., and Trowbridge, D.A.[1998]: "COMPARE - Constitutive Material Parameter Estimation, User's Guide - Version 1.0", Technical Report, Dept. of Civil Engineering, University of Akron, Akron, Ohio.

[1998b] Saleeb,A.F., Wilt, T.E., And Li, W. : "Robust Integration Schemes for Generalized Viscoplasticity with Internal-State Variables", Comp. Mech., Vol. 21, No. 6, pp. 429-440.

[2001] Saleeb, A.F., Arnold, S.M., Castelli, M.G , Wilt, T.E., And Graf, W.E.: "A General Hereditary Multimechanism-Based Deformation Model With Application to The Viscoelastoplastic Response of Titanium Alloys, Int. Jnl. of Plasticity, Vol. 17, No. 10, pp. 1305-1350.

[2002] Arsenlis, A. And Parks, D.M.; "Modeling the Evolution of Crystallographic Dislocation Density in Crystal Plasticity", Jnl. Mech. Physics of Solids, Vol. 50, pp. 19792009. 
[2002] Ho, K. And Kremple, E. : "Extension of the Viscoplasticity Theory Based on Overstress (VBO) to Capture Non-Standard Rate Dependence in Solids", Int. Jnl. of Plastcity, Vol. 18, pp. 851-872.

[2002] Saleeb, A.F., T.E. Wilt, D.A. Trowbridge, and Gendy, A.S.; "Effective Strategy for Automated Characterization in Complex Viscoelastoplastic and Damage Modeling for Isotropic/Anisotropic Aerospace Materials", Jnl of Aerospace Engineering, Vol. 15, No. 3, pp.84-96.

[2002] Stainier, L., Cuitino, A. M. And Ortiz, M.; " A Micromechanical Model of Hardening, Rate-Sensitivity and Thermal Softening in BCC Single Crystals", J. Mech. Phys. Solids, Vol. 50 , pp. 1511-1545.

[2002] ZBib, H.M. And Rubia, T.D.; "A Multiscale Model of Plasticity", Int. Jnl. of Plasticity, Vol. 18, pp. 1133-1163. 
Public reporting burden for this collection of information is estimated to average 1 hour per response, including the time for reviewing instructions, searching existing data sources, gathering and maintaining the data needed, and completing and reviewing the collection of information. Send comments regarding this burden estimate or any other aspect of this collection of information, including suggestions for reducing this burden, to Washington Headquarters Services, Directorate for Information Operations and Reports, 1215 Jefferson Davis Highway, Suite 1204, Arlington, VA 22202-4302, and to the Office of Management and Budget, Paperwork Reduction Project (0704-0188), Washington, DC 20503.

\begin{tabular}{|l|l|l}
\hline 1. AGENCY USE ONLY (Leave blank) & $\begin{array}{c}\text { 2. REPORT DATE } \\
\text { February } 2003\end{array}$ & $\begin{array}{c}\text { 3. REPORT TYPE AND DATES COVERED } \\
\text { Technical Memorandum }\end{array}$ \\
\hline
\end{tabular}

4. TITLE AND SUBTITLE

5. FUNDING NUMBERS

Specific Hardening Function Definition and Characterization of a

Multimechanism Generalized Potential-Based Viscoelastoplasticity Model

6. AUTHOR(S)

WBS-22-713-82-35

A.F. Saleeb and S.M. Arnold

7. PERFORMING ORGANIZATION NAME(S) AND ADDRESS(ES)

National Aeronautics and Space Administration

John H. Glenn Research Center at Lewis Field

Cleveland, Ohio 44135-3191

8. PERFORMING ORGANIZATION

REPORT NUMBER

E-13833

9. SPONSORING/MONITORING AGENCY NAME(S) AND ADDRESS(ES)

10. SPONSORING/MONITORING

AGENCY REPORT NUMBER

National Aeronautics and Space Administration

Washington, DC 20546-0001

NASA TM-2003-212219

\section{SUPPLEMENTARY NOTES}

A.F. Saleeb, University of Akron, Akron, Ohio 44325; and S.M. Arnold, NASA Glenn Research Center. Responsible person, S.M. Arnold, organization code 5920, 216-433-3334.

12a. DISTRIBUTION/AVAILABILITY STATEMENT

12b. DISTRIBUTION CODE

Unclassified - Unlimited

Subject Category: 39

Distribution: Nonstandard

Available electronically at http://gltrs.grc.nasa.gov

This publication is available from the NASA Center for AeroSpace Information, 301-621-0390.

13. ABSTRACT (Maximum 200 words)

Given the previous complete-potential structure framework together with the notion of strain- and stress-partitioning in terms of separate contributions of several submechanisms (viscoelastic and viscoplastic) to the thermodynamic functions (stored energy and dissipation) a detailed viscoelastoplastic multimechanism characterization of a specific hardening functional form of the model is presented and discussed. TIMETAL $21 \mathrm{~S}$ is the material of choice as a comprehensive test matrix, including creep, relaxation, constant strain-rate tension tests, etc. are available at various temperatures. Discussion of these correlations tests, together with comparisons to several other experimental results, are given to assess the performance and predictive capabilities of the present model particularly with regard to the notion of hardening saturation as well as the interaction of multiplicity of dissipative (reversible/irreversible) mechanisms.

\section{SUBJECT TERMS}

15. NUMBER OF PAGES

Viscoplasticity; Viscoelasticity; Nonlinear hardening; Isothermal; Deformation; Internal state variable; Correlations; Predictions; Multiaxial

\begin{tabular}{|c|c|}
\hline $\begin{array}{c}\text { 17. SECURITY CLASSIFICATION } \\
\text { OF REPORT } \\
\text { Unclassified }\end{array}$ & $\begin{array}{c}\text { 18. SECURITY CLASSIFICATION } \\
\text { OF THIS PAGE } \\
\text { Unclassified }\end{array}$ \\
\hline
\end{tabular}

19. SECURITY CLASSIFICATION OF ABSTRACT

Unclassified
39

16. PRICE CODE

20. LIMITATION OF ABSTRACT

Standard Form 298 (Rev. 2-89)

Prescribed by ANSI Std. Z39-18 298-102 